\title{
A Systematic Review and Meta-Analysis of the Effects of Flavanol-Containing Tea, Cocoa and Apple Products on Body Composition and Blood Lipids: Exploring the Factors Responsible for Variability in Their Efficacy
}

Antonio González-Sarrías ${ }^{1, *}$, Emilie Combet ${ }^{2}$ (D), Paula Pinto ${ }^{3}$, Pedro Mena 4 (D), Margherita Dall'Asta ${ }^{4}$, Mar Garcia-Aloy ${ }^{5,6}$ (D) , Ana Rodríguez-Mateos ${ }^{7}$, Eileen R. Gibney ${ }^{8}$ (D), Julie Dumont ${ }^{9}$, Marika Massaro ${ }^{10}$, Julio Sánchez-Meca ${ }^{11}$, Christine Morand ${ }^{12}$ and María-Teresa García-Conesa ${ }^{1, *}$ (i)

1 Research Group on Quality, Safety and Bioactivity of Plant Foods, Campus de Espinardo, Centro de Edafologia y Biologia Aplicada del Segura-Consejo Superior de Investigaciones Científicas (CEBAS-CSIC), P.O. Box 164, 30100 Murcia, Spain

2 Human Nutrition, School of Medicine, Dentistry and Nursing, College of Medical, Veterinary and Life Sciences, University of Glasgow, Glasgow G31 2ER, UK; Emilie.CombetAspray@glasgow.ac.uk

3 Polytechnic Institute of Santarem, Escola Superior Agrária (ESA), Department of Food Technology, Biotechnology and Nutrition, 2001-904 Santarém, Portugal; paula.pinto@esa.ipsantarem.pt

4 Human Nutrition Unit, Department of Food \& Drug, University of Parma, 43125 Parma, Italy; pedromiguel.menaparreno@unipr.it (P.M.); margherita.dallasta@unipr.it (M.D.)

5 Biomarkers and Nutrimetabolomic Laboratory, Department of Nutrition, Food Sciences and Gastronomy, University of Barcelona, 08028 Barcelona, Spain; margarcia@ub.edu

6 CIBER de Fragilidad y Envejecimiento Saludable (CIBERFES), Instituto de Salud Carlos III, 08028 Barcelona, Spain

7 Division of Diabetes and Nutritional Sciences, King's College London, London SE1 9NH, UK; ana.rodriguez-mateos@kcl.ac.uk

8 Institute of Food and Health, School of Agriculture and Food Science, University College Dublin (UCD), Belfield, Dublin 4, Ireland; eileen.gibney@ucd.ie

9 U1167-RID-AGE-Facteurs de risque et Déterminants Moléculaires des Maladies Liées au Vieillissement, University Lille, Institut National de la Santé et de la Recherche Médicale (INSERM), Centre Hospitalier Universitaire (CHU) Lille, Institut Pasteur de Lille, F-59000 Lille, France; julie.dumont@pasteur-lille.fr

10 National Research Council (CNR), Institute of Clinical Physiology, 73100 Lecce, Italy; marika@ifc.cnr.it

11 Department of Basic Psychology \& Methodology, Faculty of Psychology, University of Murcia, 30100 Murcia, Spain; jsmeca@um.es

12 Institut National de la Recherche Agronomique (INRA), Human Nutrition Unit, Université Clermont Auvergne (UCA), Centre de Recherches en Nutrition Humaine (CRNH) Auvergne, F-63000 Clermont-Ferrand, France; christine.morand@inra.fr

* Correspondence: agsarrias@cebas.csic.es (A.G.-S.); mtconesa@cebas.csic.es (M.-T.G.-C.); Tel.: +34-968-396276 (A.G.-S. \& M.-T.G.-C.); Fax: +34-968-396213(A.G.-S. \& M.-T.G.-C.)

Received: 26 June 2017; Accepted: 10 July 2017; Published: 13 July 2017

\begin{abstract}
Several randomized controlled trials (RCTs) and meta-analyses support the benefits of flavanols on cardiometabolic health, but the factors affecting variability in the responses to these compounds have not been properly assessed. The objectives of this meta-analysis were to systematically collect the RCTs-based-evidence of the effects of flavanol-containing tea, cocoa and apple products on selected biomarkers of cardiometabolic risk and to explore the influence of various factors on the variability in the responses to the consumption of these products. A total of 120 RCTs were selected. Despite a high heterogeneity, the intake of the flavanol-containing products was associated using a random model with changes (reported as standardized difference in means (SDM))
\end{abstract}


in body mass index $(-0.15, p<0.001)$, waist circumference $(-0.29, p<0.001)$, total-cholesterol $(-0.21$, $p<0.001)$, LDL-cholesterol $(-0.23, p<0.001)$, and triacylglycerides $(-0.11, p=0.027)$, and with an increase of HDL-cholesterol $(0.15, p=0.005)$. Through subgroup analyses, we showed the influence of baseline-BMI, sex, source/form of administration, medication and country of investigation on some of the outcome measures and suggest that flavanols may be more effective in specific subgroups such as those with a BMI $\geq 25.0 \mathrm{~kg} / \mathrm{m}^{2}$, non-medicated individuals or by specifically using tea products. This meta-analysis provides the first robust evidence of the effects induced by the consumption of flavanol-containing tea, cocoa and apple products on weight and lipid biomarkers and shows the influence of various factors that can affect their bioefficacy in humans. Of note, some of these effects are quantitatively comparable to those produced by drugs, life-style changes or other natural products. Further, RCTs in well-characterized populations are required to fully comprehend the factors affecting inter-individual responses to flavanol and thereby improve flavanols efficacy in the prevention of cardiometabolic disorders.

Keywords: flavanols; tea; cocoa; apple; cardiometabolic disorders; meta-analysis; interindividual variability; blood lipids; body mass index; waist circumference

\section{Introduction}

Metabolic disorders, principally, abdominal obesity, dyslipidemia (high levels of triacylglycerides (TAGs) and low levels of high-density lipoprotein (HDL)), and insulin resistance have been associated to an increased risk of Type-2 diabetes mellitus (DM) and cardiovascular diseases (CVDs). CVDs remain the number one cause of death in developed countries and their prevalence is increasing rapidly in developing nations and in adolescents [1]. It is now well established from population studies that some aspects of CVDs risk can be modulated by various dietary interventions including an increased consumption of plant foods [2], as part of a healthy balanced diet. In addition to other protective compounds (i.e., fiber and vitamins), plant foods are an exclusive and abundant source of phytochemicals, a large and diverse group of compounds which exhibit an array of biological activities. The intake of these bioactive compounds are thought to contribute to the health benefits associated with the consumption of such foods [3]. Polyphenols are some of the most abundant phytochemicals in plant foods and increasing evidence from cohort studies indicate that the intake of some of these compounds such as diverse flavonoids and/or, importantly, some of their derived microbial metabolites (e.g., enterolactone) may help to reduce the development of CVDs and CVDs mortality risk [4-7]. This evidence is supported by animal and clinical studies reporting beneficial effects of the consumption of some polyphenol-rich foods or pure compounds on CVDs risk factors such as blood cholesterol, blood pressure, endothelial function and arterial stiffness [8].

Polyphenols encompass several families of compounds, the most represented in plant foods being phenolic acids and flavonoids [9]. A major group of flavonoids is constituted by flavanols that are abundant in green tea, red wine, cocoa and various fruits such as apples [10]. A summary of the major flavonoids present in tea (green and black), cocoa powder and apple is shown in Table S1. The assessment of daily intakes of flavanols across Europe revealed a large variation between countries (from 200 to $800 \mathrm{mg} /$ day) depending on their dietary habits and the intake of tea [11]. The flavanol group is composed primarily of the epicatechin and catechin monomers, and of their oligomeric and polymeric forms, the procyanidins. Flavanol monomers and dimeric procyanidins are bioavailable. They undergo extensive phase II conjugation and are found in the blood circulation mostly as $O$-methylated, sulfated and glucuronidated conjugates (nM to $\mu \mathrm{M}$ range) [12,13]. In contrast, the procyanidins polymers are not absorbed and do not contribute to the systemic pool of flavanols in humans [14]. 
Human studies remain essential to understanding the effects of the plant bioactive compounds on health and thus, an increasing number of randomized controlled trials (RCTs) with flavanol-containing products have been carried out over the past two decades. Meta-analyses constitute a useful tool to integrate the accumulated RCTs and review the evidence in humans. Some of the main problems affecting the results of meta-analyses are the usually limited number of studies included as well as a range of factors that introduce heterogeneity in the findings. Identifying the factors underlying variability, as well as developing new and innovative methodologies to account for such variability constitute an overarching goal to ultimately optimize the beneficial health effects of plant food bioactives. Among the potential factors involved in such heterogeneity are: (i) factors inherent to the individuals: (epi) genetic factors, gut microbiota, baseline conditions (BMI, medication), sex, health status, ethnicity, and age; and (ii) factors intrinsic to the type of study (design, duration, dose, and type of product) [15].

The main goals of the present study were: (i) to systematically review and appraise, through meta-analysis, the impact of flavanol-containing tea, cocoa and apple products, three main sources of flavanols, on selected biomarkers of cardiometabolic risk, i.e., BMI, WC and blood lipid levels (total-, LDL-, HDL-cholesterol and TAGs); and (ii) to further explore some of the factors that may be implicated in the inter-individual variability in the response to the consumption of these flavanol-containing products.

\section{Materials and Methods}

This systematic review and meta-analysis followed the PRISMA (Preferred Reporting Items for Systematic Reviews and Meta-Analyses) statement guidelines [16], the Cochrane Handbook for Systematic Reviews of Interventions [17], and the Centre for Reviews and Dissemination's guidance for undertaking reviews in health care [18]. The protocol for this review was registered in the International Prospective Register of Systematic Reviews (PROSPERO, www.crd.york.ac.uk/prospero/index.asp) with the registration number CRD42016033878.

\subsection{Search Strategy}

A comprehensive search on PubMed and Web of Science databases was conducted in July 2015. Search terms included a combination of keywords referring to: (1) bioactive (polyphenols, flavonoids, flavanols, flavan-3-ol, (epi)catechin, (epi)gallocatechin gallate, theaflavins, thearubigin, and procyanidin); (2) food source (apple, tea, and cocoa); (3) type of study and participants (trial, experiment, study, intervention; human, subjects, men, women, patients, volunteers, and participants); and (4) cardiometabolic outcomes (BMI, WC, total cholesterol, LDL cholesterol, HDL cholesterol, and TAGs). No type of restriction was applied during the electronic searches.

\subsection{Study Selection and Data Extraction}

Two authors independently assessed all papers and in the case of disagreement, discussed findings to reach a consensus, or in the absence of resolution, a third author was contacted. Studies included in the meta-analysis were limited to human RCTs testing the effect of flavanol-containing tea, cocoa or apple products, which had a control group receiving a placebo and measured one or more of the defined outcomes (BMI, WC, total cholesterol, LDL cholesterol, HDL cholesterol, or TAGs). Manuscripts written in any European language were included, whereas other manuscripts were excluded. Additionally, the studies with the following characteristics were excluded: studies with flavanol-rich food sources other than tea, cocoa or apples; and studies with multifactorial interventions (i.e., flavanols given as a part of a multicomponent treatment; dietary or physical activity co-intervention). Data extraction was performed in duplicate by two authors, independently, and cross-checked by a third author using a standardized data extraction form. Extracted data included publication details (year of publication, contact details, clinical trial and registration number); participant characteristics (geographical origin, total number of participants included in the study and in the analysis, sex distribution, age, ethnicity, 
health status, menopausal status, smoking habits, and use of medication); study setting and design (cross-over or parallel design, duration of the intervention, number of arms and description, number of participants located in each arm and completing the study, composition of test and placebo, and dose and mode of administration); and outcomes (type of sample, changes in the outcome, values before and after intervention, and $p$-value).

\subsection{Assessment of Quality and Data Analysis}

The quality of the studies was assessed based on the Cochrane Collaboration measurement with some modifications [19]. The specific items used for the assessments are detailed in a previous meta-analysis following the same protocol [7].

Data for each outcome were analyzed using the Comprehensive Meta-Analysis Software, version 3.0 (Biostat, Englewood, NJ, USA) [20]. The free scale index standardized difference in means (SDM) was used to combine data from the highest number of collected valid studies, increasing the pool of studies and the power to detect significant differences. SDM, standard error (SE) and the corresponding $95 \%$ confidence intervals (CI) were calculated and pooled using random effects models to determine test/placebo differences across studies. Statistical heterogeneity between studies was assessed by using the Cochran $Q$ test, the between-studies variance $\left(T^{2}\right)$ and $I^{2}$ (an estimate of the proportion of variance across studies caused by heterogeneity rather than by random errors) where $I^{2}$ values equal to $25 \%, 50 \%$ and $75 \%$ were considered as low, moderate and high heterogeneity, respectively. Publication bias was assessed visually with funnel plots and statistically by applying the Egger's regression test. Further assessment of the possible associations between the overall changes attributed to the supplementation with the flavanols and the duration of the intervention was examined using random-effects meta-regression analysis. Using the random model, we have additionally estimated the overall effect size as the difference in means (DM) and 95\% CI.

Subgroup analyses were conducted to explore potential factors that may introduce heterogeneity into the studies and influence the inter-individual variability in the response to supplementation with the flavanol-containing products. We selected those factors that were more clearly described throughout articles (Table 1). We included factors that might be attributed to some of the individuals' characteristics, such as baseline BMI, sex, smoking habits and medication/health status. Age or ethnicity could not be assessed due to unclear reporting. We also included stratification by the country in which the study was carried out, the source and form of administration of the flavanols, as well as the type of diet reported to be followed during the intervention. For each subgroup, the pooled effects (SDM) and the significance of this value were estimated. Additionally, statistical comparisons between subgroups were performed by applying a random-effects analysis and calculation of the between-categories $\mathrm{Q}$ statistic, the $p$-value and the $\mathrm{R}^{2}$ index (proportion of between-studies variance explained by each factor or covariate). Using some of the factors that partially explained some of the between-studies variance for a particular variable, we applied a multiple meta-regression analysis with a random-effects model to search for a potential combination of factors that best explained the between-study variance for this variable. Statistical significance of the findings was as follows: $p$-value $<0.05$ was considered significant, while $p$-value $\geq 0.05$ and $<0.1$ was considered marginally significant. 
Table 1. Potential factors influencing the heterogeneity in the responses to the supplementation with flavanols-containing products investigated in this meta-analysis.

\begin{tabular}{|c|c|c|c|c|c|c|}
\hline \multicolumn{7}{|l|}{ Factors } \\
\hline Baseline BMI & \multicolumn{3}{|c|}{$<25.0^{\text {a }}$ (normal and/or underweight) } & \multicolumn{3}{|c|}{$\geq 25.0$ (overweight and/or obese) } \\
\hline Sex & \multicolumn{3}{|c|}{ Women } & \multicolumn{3}{|c|}{ Men } \\
\hline Smoking & \multicolumn{3}{|c|}{ Non-smokers } & \multicolumn{3}{|c|}{ Smokers } \\
\hline \multirow[b]{2}{*}{$\begin{array}{l}\text { Country where } \\
\text { the study was } \\
\text { undertaken }\end{array}$} & \multirow[b]{2}{*}{$\begin{array}{l}\text { East Asian } \\
\text { countries (Japan, } \\
\text { Korea, China, } \\
\text { Thailand, } \\
\text { Taiwan) }\end{array}$} & \multirow[b]{2}{*}{$\begin{array}{l}\text { All other } \\
\text { countries }\end{array}$} & \multirow[b]{2}{*}{$\begin{array}{l}\text { North America } \\
\text { (USA, Canada) }\end{array}$} & \multicolumn{3}{|c|}{ European countries } \\
\hline & & & & $\begin{array}{l}\text { Non-Mediterranean countries } \\
\text { (Denmark, Finland, The } \\
\text { Netherlands, Germany, } \\
\text { Poland, UK, Switzerland) }\end{array}$ & $\begin{array}{l}\text { Mediter } \\
\text { (Italy, }\end{array}$ & $\begin{array}{l}\text { countries } \\
\text { Portugal, } \\
\text { e) }\end{array}$ \\
\hline Medication & \multicolumn{3}{|c|}{ Yes } & \multicolumn{3}{|l|}{ No } \\
\hline $\begin{array}{l}\text { Healthy vs. } \\
\text { non-healthy }\end{array}$ & $\begin{array}{l}\text { Healthy } \\
\text { individuals } b\end{array}$ & $\begin{array}{l}\text { Individuals } \\
\text { at a risk of } \\
\text { disease }^{c}\end{array}$ & $\begin{array}{l}\text { Individuals with } \\
\text { a reported } \\
\text { disease }^{\mathrm{d}}\end{array}$ & \multicolumn{3}{|c|}{$\begin{array}{c}\text { Different disorders: overweight and/or obese, } \\
\text { dyslipidemia, glucose disorders, blood pressure disorders, } \\
\text { mixed }{ }^{\text {e }}\end{array}$} \\
\hline $\begin{array}{l}\text { Source of } \\
\text { flavanols }\end{array}$ & Cocoa products & $\begin{array}{l}\text { Apple } \\
\text { products }\end{array}$ & Tea products & $\begin{array}{r}\text { Tea extracts ( } \\
\text { powde }\end{array}$ & apsules, & $\begin{array}{l}\text { Tea } \\
\text { purified } \\
\text { EGCG }\end{array}$ \\
\hline $\begin{array}{l}\text { Diet during } \\
\text { intervention }\end{array}$ & \multicolumn{3}{|c|}{ Controlled diet } & \multicolumn{3}{|c|}{$\begin{array}{l}\text { Usual diet } \\
\text { (includes usual with some restrictions and NR) }\end{array}$} \\
\hline
\end{tabular}

${ }^{a}$ : BMI cut-off values as established by the $\mathrm{WHO}^{\mathrm{b}}$ : Includes individuals specifically reported as healthy and not medicated (in some cases medication was not reported, NR); ${ }^{c}$ : Includes individuals not medicated that were overweight and/or obese, or specifically indicated to be borderline, mild condition or at risk of a disease; ${ }^{\mathrm{d}}$ : Includes individuals with one or more than one of the following disorders: dyslipidemia, glucose disorders or type-2 diabetes, blood pressure disorders (hypertension), medicated obesity, metabolic syndrome (most cases were also medicated but in some cases medication was NR); ${ }^{\text {e }}$ Individuals reported to have only one of the specified disorders.

\section{Results}

\subsection{Description of the Included Studies}

A total of 1409 articles were initially identified through the search on the electronic databases. After removal of duplicates and screening, 188 trials were selected for data extraction. After detailed analysis of the full text, 71 articles were excluded, due to lack of relevant outcomes, aspects of study design or publication language. The final number of articles selected for meta-analysis was selected from a total of 117 articles published between 1997 and July 2015 (included) [21-137]. The detailed study selection flow diagram is shown in Figure 1.

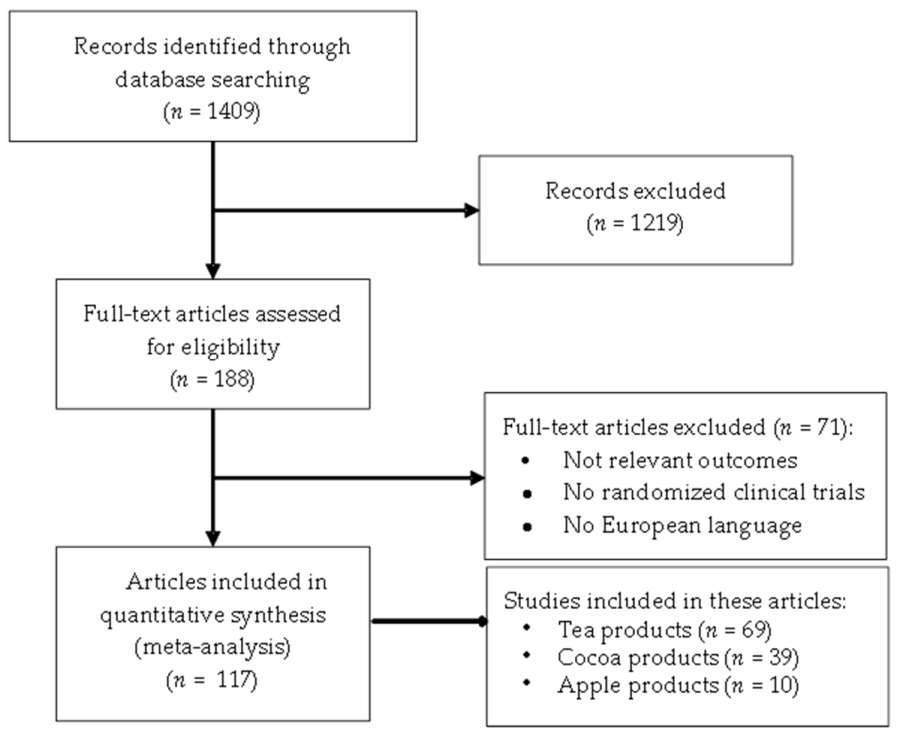

Figure 1. Flow diagram showing the study selection process. 


\subsection{Quality and Characteristics of the Selected Studies}

Most of the studies (70\%) were classified as studies with a moderate to low risk of bias (quality score $\geq 5.0$ and $<8.0$ or $\geq 8.0$ and $\leq 10.0$, respectively) while $30 \%$ of the studies obtained a low quality score $(<5.0)$ and were considered as a high risk of bias.

The studies were carried out in countries distributed over five continents: Asia (Japan, Korea, China, Taiwan, Thailand, Saudi Arabia, and Iran), North America (USA and Canada) and Latin America (Brazil and Mexico), Europe (Denmark, Finland, The Netherlands, Germany, Poland, UK, Switzerland, Italy, Spain, Portugal, and Greece), Africa (Mauritania, South Africa, and Republic of Mauritius), and Australia. Thus, they were considered representative of a global population. The participants in these studies also represent a mixed population of men and women ranging from young adults to elderly participants, and with a higher prevalence of individuals with a BMI $\geq 25.0 \mathrm{~kg} / \mathrm{m}^{2}$ (overweight and/or obese volunteers). The quality and depth of reporting of the factors potentially contributing toward inter-individual variability of the effect of flavanols varied among studies. The smoking habits were not reported in most studies but for those studies that did, the participants were typically non-smokers or a mixed sample population. Only two studies [34,57] were carried out specifically with smokers. The total sample population included healthy individuals, overweight and/or obese individuals as well as individuals with an incipient or with a reported chronic risk factor or metabolic disease, comprising principally hypertension, hyperlipidemias, type- 2 diabetes, metabolic syndrome, atherosclerosis, coronary artery disease and heart failure. Among these, some participants were taking medication, others were not medicated or medication use was not reported. Studies were selected if the source of flavanols was tea, cocoa, or apple provided as liquid (tea drinks, cocoa beverages, and apple juice) or solid (powder or extracts in capsules, snacks, tablets, and foods) forms. Interventions ranged typically 1-6 months, during which participants followed either a controlled diet or their habitual diets.

\subsection{Overall Impact of the Supplementation with Flavanol-Containing Tea, Cocoa or Apple Products on Blood} Lipids, BMI and WC

The number of RCTs varied in function of the outcome measure studied, from 46 to 120 trials, recruiting a total high number of participants ranging from 2875 to 5931 individuals. Forest plots detailing weighted SDM, SE, 95\% confidence intervals and relative weight for the impact of supplementation with flavanol-containing tea, cocoa or apple products on BMI, WC, and blood lipid levels are shown in Figures S1-S6. Visual inspection of the Funnel plots (Figures S7-S12) evidenced symmetrical shapes and absence of publication bias in the case of WC, total cholesterol, LDL, HDL and TAGs. Some asymmetry was however detected for BMI. These results were further confirmed by Egger's regression. A summary of the random overall effects for each lipid and obesity-related variable, heterogeneity and bias analyses is presented in Table 2.

Table 2. Overall changes (SDM), heterogeneity and publication bias analyses for the impact of flavanol-containing products on BMI, WC and blood lipids levels.

\begin{tabular}{|c|c|c|c|c|c|c|c|c|c|c|c|c|c|c|}
\hline & \multirow[b]{2}{*}{$n$} & \multirow[b]{2}{*}{$\mathbf{N}_{\mathrm{T}}$} & \multirow[b]{2}{*}{$\mathbf{N}_{\mathrm{S}}$} & \multirow[b]{2}{*}{$\mathbf{N}_{\mathrm{C}}$} & \multirow[b]{2}{*}{ SDM } & \multirow[b]{2}{*}{$95 \%$ CI } & \multirow[b]{2}{*}{$\mathbf{Z}$} & \multirow[b]{2}{*}{$p$-Value } & \multirow[b]{2}{*}{$\mathrm{Tau}^{2}$} & \multirow[b]{2}{*}{ Q } & \multirow[b]{2}{*}{$d f$ ( $p$-Value) } & \multirow[b]{2}{*}{$I^{2}(\%)$} & \multicolumn{2}{|c|}{ Egger's Regression } \\
\hline & & & & & & & & & & & & & Intercept & $\begin{array}{c}p \text {-Value } \\
\text { (2-Tailed) }\end{array}$ \\
\hline BMI & 74 & 4156 & 2127 & 2029 & -0.153 & $-0.227,-0.078$ & -4.009 & $<0.001$ & 0.027 & 99.6 & $73(0.021)$ & 26.7 & 1.04 & 0.024 \\
\hline WC & 46 & 2875 & 1478 & 1397 & -0.293 & $-0.438,-0.147$ & -3.932 & $<0.001$ & 0.168 & 156.7 & $45(<0.001)$ & 71.3 & -1.47 & 0.101 \\
\hline TC & 112 & 5812 & 2982 & 2830 & -0.214 & $-0.328,-0.099$ & -3.651 & $<0.001$ & 0.273 & 479.1 & $111(<0.001)$ & 76.8 & -0.76 & 0.226 \\
\hline LDL-C & 105 & 5726 & 2928 & 2798 & -0.235 & $-0.345,-0.125$ & -4.171 & $<0.001$ & 0.229 & 408.0 & $104(<0.001)$ & 74.5 & -0.46 & 0.459 \\
\hline HDL-C & 112 & 5928 & 3023 & 2905 & 0.152 & $0.047,0.256$ & 2.836 & 0.005 & 0.214 & 408.0 & $111(<0.001)$ & 72.8 & 0.72 & 0.208 \\
\hline TAGs & 120 & 5931 & 3023 & 2908 & -0.114 & $-0.215,-0.013$ & -2.213 & 0.027 & 0.209 & 407.7 & $119(<0.001)$ & 70.8 & -0.33 & 0.570 \\
\hline
\end{tabular}

BMI: Body Mass Index; WC: Waist Circumference; TC: Total Cholesterol; LDL-C: Low density Lipoprotein Cholesterol; HDL-C: High Density Lipoprotein Cholesterol; TAGs: Triglycerides; $n$ : total number of studies included in the analysis; $\mathrm{N}_{\mathrm{T}}$ : number of total participants; $\mathrm{N}_{\mathrm{S}}$ : number of participants in the supplemented group; $\mathrm{N}_{\mathrm{C}}$ : number of participants in the control group; SDM: standardized difference in means; $95 \%$ CI: lower and upper confidence limits for the average SDM: $d f$ : degrees of freedom; Z: statistic for testing the significance of the average $\mathrm{SDM}$; $\mathrm{Tau}^{2}$ : between-studies variance; $\mathrm{Q}$ : heterogeneity statistic; $\mathrm{I}^{2}$ : heterogeneity index. 
Despite a high heterogeneity across the studies $\left(I^{2}=70-77 \%\right.$ for most variables except for BMI which was more moderate, $I^{2}=26.7 \%$ ), the overall pooled analysis (shown as SDM) significantly confirmed a reduction of BMI $(-0.153, p$-value $<0.001)$, WC $(-0.293, p$-value $<0.001)$, blood total cholesterol $(-0.214, p$-value $<0.001)$, LDL $(-0.235, p$-value $<0.001)$, and TAGs $(-0.114, p$-value $=0.027)$. HDL levels were also significantly increased $(0.152, p$-value $=0.005)$. Sensitivity analyses were carried out using the leave-one-out approach where the meta-analysis was performed with each study removed in turn. The pooled estimates consistently showed a similar effect and significance emphasizing the robustness of these results and that the effect was not driven by any particular study (data not shown). Further support of these results was found by a significant relationship between the duration of the supplementation with the flavanol products and the reduction of WC; total-, LDL- and HDL-cholesterol; and TAGs using random-effects meta-regression analysis. Regression coefficients and $p$-values for each variable can be seen in Table S2.

\subsection{Analysis of the Potential Factors Influencing Inter-Individual Responses to Flavanols Consumption}

\subsubsection{Stratification by the Individuals' Baseline BMI, Sex, Smoking, and Country}

Following stratification by the baseline BMI (Table 3), the effects of the flavanol-containing products on BMI, WC, total- and LDL-cholesterol remained significant only in those studies carried out in overweight/obese volunteers (BMI $\geq 25.0 \mathrm{~kg} / \mathrm{m}^{2}$ ). HDL-cholesterol levels were also increased in this subgroup $(p=0.063)$ whereas the reducing effects on TAGs levels were not significant in any of the two subgroups. Statistical comparison between $\geq 25.0 \mathrm{vs.}<25.0 \mathrm{~kg} / \mathrm{m}^{2}$ subgroups did not reach significance for any of the variables investigated. Of note, $61 \%$ of the total between-study variance in BMI could be explained by the respective baseline BMI values (total between $Q=2.53, p$-value $=0.112, R^{2}$ index $=0.61$ ).

Regarding stratification by sex, the reduction of WC was significant in both men and women after intervention with flavanol-containing products. However, total- and LDL-cholesterol were significantly reduced only in female whereas BMI was significantly lowered only in male. The effects on HDL and TAG levels were no longer significant after stratification by sex (Table 3). Between groups comparison indicated a difference between sexes (total between $\mathrm{Q}=2.833, p$-value $=0.092$ ) and a considerable contribution of the sex to the between-study variance for BMI $\left(\mathrm{R}^{2}\right.$ index $\left.=1.0\right)$.

The reduction of BMI, WC, and total- and LDL-cholesterol in response to the flavanol-containing products was significant in studies carried out in non-smoker volunteers. It was not possible, however, to establish a comparison with habitual smokers due to the very low number of studies carried out with this type of volunteers ( $n=2$ studies).

Comparison between studies carried out in East Asian countries (assuming Asian ethnicity) against those carried out elsewhere evidenced similar results for BMI, WC, total and LDL cholesterol in both subgroups although the results were slightly less significant in the East Asian subgroup. A small proportion (7\%) of the BMI between-groups variance was explained by the study location (East Asian vs. others) (total between $Q=0.963, p$-value $=0.327, R^{2}$ index $=0.07$ ). In addition, we found a significant difference in TAG levels in response to flavanol-containing products between the East Asian subgroup and the others with a more pronounced effect in the East Asian studies (total between $\mathrm{Q}=7.419, p$-value $=0.024, \mathrm{R}^{2}$ index $<0.01$ ). When grouping in North America and European countries, we detected a significant reduction of BMI and WC in the Europe group but not in the American one, whereas the LDL-cholesterol reduction resulted significant in the American group only. Statistical comparison between North America and Europe groups showed a difference in the BMI response (total between $\mathrm{Q}=3.143$, $p$-value $=0.076$ ) and a $28 \%$ of the between-group variance explained by this factor $\left(R^{2}\right.$ index $\left.=0.28\right)$. Within Europe, the studies carried out in countries of the Mediterranean area resulted in significant reductions of BMI, WC, total- and LDL-cholesterol and in an increase of HDL $(p=0.078)$. In the non-Mediterranean countries, we only detected a significant reduction of total-cholesterol. Comparison between the two subgroups indicated a significant difference in the WC reduction (Total between $Q=5.228, p$-value $=0.022, R^{2}$ index $<0.01$ ). 
Table 3. Stratification analysis of the influence of baseline BMI, sex, smoking and country where the study was carried out on the effects (SDM) on BMI, WC, and blood lipids levels following supplementation with flavanol-containing products.

\begin{tabular}{|c|c|c|c|c|c|c|c|c|c|c|c|c|}
\hline \multirow{3}{*}{$\begin{array}{c}\text { Factor } \\
\text { Subgroup }\end{array}$} & \multicolumn{2}{|c|}{ Baseline BMI } & \multicolumn{2}{|c|}{ Sex } & \multicolumn{2}{|c|}{ Smoking } & \multicolumn{6}{|c|}{ Country Where the Study Was Undertaken } \\
\hline & \multirow{2}{*}{$<25.0$} & \multirow{2}{*}{$\geq 25.0$} & \multirow{2}{*}{ Women } & \multirow{2}{*}{ Men } & \multirow{2}{*}{ Non-Smokers } & \multirow{2}{*}{ Smokers } & \multirow{2}{*}{$\begin{array}{l}\text { East Asian } \\
\text { Countries }\end{array}$} & \multirow{2}{*}{$\begin{array}{l}\text { All Other } \\
\text { Countries }\end{array}$} & \multirow{2}{*}{$\begin{array}{c}\text { North } \\
\text { America }\end{array}$} & \multirow{2}{*}{$\begin{array}{l}\text { All European } \\
\text { Countries }\end{array}$} & \multicolumn{2}{|c|}{ European Countries } \\
\hline & & & & & & & & & & & $\begin{array}{c}\text { Med } \\
\text { Countries }\end{array}$ & $\begin{array}{l}\text { Non-Med } \\
\text { Countries }\end{array}$ \\
\hline $\begin{array}{c}\text { BMI } \\
p \text {-value } \\
(n, \mathrm{~N})\end{array}$ & $\begin{array}{c}0.003^{\mathrm{a}} \\
(\mathrm{NS}) \\
(11,477)\end{array}$ & $\begin{array}{c}-0.165 \\
(0.001) \\
(42,2410)\end{array}$ & $\begin{array}{c}-0.102 \\
(\mathrm{NS}) \\
(15,831)\end{array}$ & $\begin{array}{l}-0.321 \\
(0.003) \\
(8,339)\end{array}$ & $\begin{array}{c}-0.148 \\
(0.018) \\
(24,1046)\end{array}$ & NR & $\begin{array}{c}-0.204 \\
(0.001) \\
(23,1511)\end{array}$ & $\begin{array}{c}-0.126 \\
(0.007) \\
(51,2645)\end{array}$ & $\begin{array}{c}-0.042 \\
(\mathrm{NS}) \\
(15,719)\end{array}$ & $\begin{array}{c}-0.235 \\
(0.001) \\
(20,1051)\end{array}$ & $\begin{array}{l}-0.306 \\
(0.024) \\
(7,392)\end{array}$ & $\begin{array}{c}-0.176 \\
(0.091) \\
(13,659)\end{array}$ \\
\hline $\begin{array}{c}\text { WC } \\
p \text {-value } \\
(n, \mathrm{~N})\end{array}$ & $\begin{array}{c}-0.102 \\
(\mathrm{NS}) \\
(3,253)\end{array}$ & $\begin{array}{c}-0.362 \\
(0.000) \\
(36,2002)\end{array}$ & $\begin{array}{l}-0.643 \\
(0.037) \\
(9,464)\end{array}$ & $\begin{array}{l}-0.932 \\
(0.024) \\
(5,266)\end{array}$ & $\begin{array}{c}-0.252 \\
(0.008) \\
(16,750)\end{array}$ & NR & $\begin{array}{c}-0.217 \\
(0.055) \\
(19,1458)\end{array}$ & $\begin{array}{c}-0.355 \\
(0.000) \\
(27,1471)\end{array}$ & $\begin{array}{c}-0.123 \\
(\mathrm{NS}) \\
(6,206)\end{array}$ & $\begin{array}{c}-0.573 \\
(0.006) \\
(13,757)\end{array}$ & $\begin{array}{l}-1.279 \\
(0.001) \\
(5,269)\end{array}$ & $\begin{array}{c}-0.181 \\
(\mathrm{NS}) \\
(8,488)\end{array}$ \\
\hline $\begin{array}{c}\text { TC } \\
p \text {-value } \\
(n, \mathrm{~N})\end{array}$ & $\begin{array}{c}-0.047 \\
(\mathrm{NS}) \\
(19,504)\end{array}$ & $\begin{array}{c}-0.165 \\
(0.011) \\
(50,2863)\end{array}$ & $\begin{array}{c}-0.493 \\
(0.012) \\
(15,873)\end{array}$ & $\begin{array}{c}-0.100 \\
(\mathrm{NS}) \\
(14,557)\end{array}$ & $\begin{array}{c}-0.221 \\
(0.001) \\
(41,1572)\end{array}$ & $\begin{array}{c}0.147 \\
\text { (NS) } \\
(2,88)\end{array}$ & $\begin{array}{c}-0.195 \\
(0.054) \\
(37,2297)\end{array}$ & $\begin{array}{c}-0.223 \\
(0.002) \\
(75,3515)\end{array}$ & $\begin{array}{c}-0.303 \\
(0.027) \\
(23,1097)\end{array}$ & $\begin{array}{c}-0.254 \\
(0.032) \\
(30,1296)\end{array}$ & $\begin{array}{c}-0.255 \\
(0.003) \\
(15,609)\end{array}$ & $\begin{array}{c}-0.252 \\
(0.003) \\
(15,639)\end{array}$ \\
\hline $\begin{array}{c}\text { LDL-C } \\
p \text {-value } \\
(n, \mathrm{~N})\end{array}$ & $\begin{array}{c}-0.062 \\
(\mathrm{NS}) \\
(13,396)\end{array}$ & $\begin{array}{c}-0.195 \\
(0.002) \\
(48,2698) \\
\end{array}$ & $\begin{array}{c}-0.545 \\
(0.004) \\
(15,868)\end{array}$ & $\begin{array}{c}-0.131 \\
(\mathrm{NS}) \\
(10,416)\end{array}$ & $\begin{array}{c}-0.156 \\
(0.039) \\
(42,1764) \\
\end{array}$ & $\begin{array}{c}-0.341 \\
(\mathrm{NS}) \\
(2,88)\end{array}$ & $\begin{array}{c}-0.236 \\
(0.017) \\
(31,2334) \\
\end{array}$ & $\begin{array}{c}-0.234 \\
(0.001) \\
(74,3392) \\
\end{array}$ & $\begin{array}{c}-0.379 \\
(0.006) \\
(25,1117) \\
\end{array}$ & $\begin{array}{c}-0.185 \\
(\mathrm{NS}) \\
(27,1176)\end{array}$ & $\begin{array}{c}-0.246 \\
(0.042) \\
(12,537) \\
\end{array}$ & $\begin{array}{c}-0.140 \\
\text { (NS) } \\
(15,639)\end{array}$ \\
\hline $\begin{array}{c}\text { HDL-C } \\
p \text {-value } \\
(n, \mathrm{~N})\end{array}$ & $\begin{array}{c}0.229 \\
\text { (NS) } \\
(17,455)\end{array}$ & $\begin{array}{c}0.176 \\
(0.063) \\
(52,2872)\end{array}$ & $\begin{array}{c}0.226 \\
\text { (NS) } \\
(15,902)\end{array}$ & $\begin{array}{c}0.272 \\
\text { (NS) } \\
(16,634)\end{array}$ & $\begin{array}{c}0.090 \\
(\mathrm{NS}) \\
(45,1945)\end{array}$ & $\begin{array}{c}0.152 \\
(\mathrm{NS}) \\
(2,88)\end{array}$ & $\begin{array}{c}0.105 \\
(\mathrm{NS}) \\
(37,2410)\end{array}$ & $\begin{array}{c}0.176 \\
(0.008) \\
(75,3518)\end{array}$ & $\begin{array}{c}0.286 \\
(0.095) \\
(22,978)\end{array}$ & $\begin{array}{c}0.165 \\
\text { (NS) } \\
(34,1495)\end{array}$ & $\begin{array}{c}0.320 \\
(0.078) \\
(15,679)\end{array}$ & $\begin{array}{c}0.029 \\
(\mathrm{NS}) \\
(18,816)\end{array}$ \\
\hline $\begin{array}{c}\text { TAGs } \\
\text { p-value } \\
(n, \mathrm{~N})\end{array}$ & $\begin{array}{c}-0.058 \\
(\mathrm{NS}) \\
(23,596)\end{array}$ & $\begin{array}{c}-0.098 \\
(\mathrm{NS}) \\
(53,2599)\end{array}$ & $\begin{array}{c}-0.067 \\
(\mathrm{NS}) \\
(16,887)\end{array}$ & $\begin{array}{c}0.109 \\
(\mathrm{NS}) \\
(18,781)\end{array}$ & $\begin{array}{c}-0.051 \\
(\mathrm{NS}) \\
(55,2243)\end{array}$ & $\begin{array}{c}0.089 \\
\text { (NS) } \\
(2,88)\end{array}$ & $\begin{array}{c}-0.196 \\
(0.034) \\
(35,1975)\end{array}$ & $\begin{array}{c}-0.052 \\
(\mathrm{NS}) \\
(83,3796)\end{array}$ & $\begin{array}{c}-0.098 \\
(\mathrm{NS}) \\
(23,921)\end{array}$ & $\begin{array}{c}-0.185 \\
(0.099) \\
(41,1969)\end{array}$ & $\begin{array}{c}-0.161 \\
(\mathrm{NS}) \\
(18,939)\end{array}$ & $\begin{array}{c}-0.203 \\
(\mathrm{NS}) \\
(24,1100)\end{array}$ \\
\hline
\end{tabular}

${ }^{a}$ Standardized difference in means (SDM); BMI: Body Mass Index; WC: Waist Circumference; TC: Total Cholesterol; LDL-C: Low density Lipoprotein Cholesterol; HDL-C: High Density Lipoprotein Cholesterol; TAGs: Triacylglycerides; Med: Mediterranean; $p$-value $<0.05$ was considered significant ; $p$-value $<0.1$ and $\geq 0.05$ was considered marginally significant ; NS: No significant change/effect; $(n)$ : Number of studies included; $(\mathrm{N})$ : Total number of participants; NR: Not reported. 


\subsubsection{Stratification by the Individuals' Medication and Health/Disease Status}

The influence of medication on the response to the consumption of the flavanol-containing products was also explored (Table 4). The subgroup including participants without any reported medication showed significant reductions of BMI, WC, total- and LDL-cholesterol as well as a reduction of TAGs $(p=0.063)$. In contrast, in the subgroup of studies including participants under medication the effects did not reach statistical significance. Further comparison between the two subgroups (Yes vs. No medication) revealed no significant differences between them (total between $Q=2.59$, $p$-value $=0.107$ ) but $34 \%$ of the between-groups variance for the BMI response was explained by this factor $\left(R^{2}\right.$ index $\left.=0.34\right)$.

Regarding health/disease status, participants were stratified as healthy, at risk or with a reported disease. Both in healthy subjects and in participants with a disease, the total- and LDL-cholesterol levels were significantly reduced. Studies conducted with volunteers categorized as at a risk exhibited the most significant reduction of BMI and WC in response to the flavanols. Stratification of the studies by the type of disorder showed a significant reduction of BMI and WC in overweight and/or obese individuals, a significant increase of HDL-cholesterol levels in patients with a dyslipidemia and a significant reduction of LDL-cholesterol in patients with diabetes or hypertension (Table 4). Comparison between each of the subgroups against the healthy subgroup was not significant for any of the variables investigated.

\subsubsection{Stratification by the Source/Administration Form of the Flavanols and the Diet during} the Intervention

Among the sources of flavanols investigated, our meta-analysis confirmed that supplementation with tea derived products significantly impacts on all the investigated variables except for TAGs (Table 5). Studies carried out with cocoa as the source of flavanols exhibited a significant effect on total-, LDL-cholesterol and TAGs levels whereas intervention with the apple-derived products appears to only modulate total- and LDL-cholesterol levels. Statistical comparison between the sources of flavanols highlighted a significant difference in the effect on BMI between tea and cocoa products ( $p$-value $=0.012$ ) with a $29 \%$ of the between-groups variance explained by this factor $\left(R^{2}\right.$ index $=0.29$ ). The apple group resulted also significantly more efficient than the cocoa or tea groups in the reduction of total-cholesterol. In addition, the apple products showed a greater effect on LDL-cholesterol than the tea derived products.

Regarding the supplementation form, the results showed that the administration of tea as solid extracts caused a significant and efficient modulation of all the variables investigated except for HDL and TAGs, whereas the tea beverages were significant at reducing only BMI and LDL-cholesterol (Table 5). Statistical comparison between liquid and solid tea-flavanols administration pointed out at a difference between the two subgroups at reducing LDL cholesterol ( $p$-value $=0.096$ ) with $11 \%$ of the between-group variance explained by this factor $\left(R^{2}\right.$ index $\left.=0.11\right)$. A very limited number of studies have reported so far the effects of tea purified epigallocatechin gallate (EGCG), one of the main flavanols present in tea. Overall, these studies only support a significant reduction of BMI by this compound. Of note, and as opposed to tea products, the purified EGCG appears to reduce the levels of HDL (results not significant) and increase those of TAGs $(p=0.077)$ (Table 5). Comparison between the EGCG subgroup and the tea drink or the tea extract subgroups indicated that the form of administration (as a purified compound or as a mixture) partially contributed to explaining the between groups variances for HDL (total between $Q=5.211$, $p$-value $=0.022, R^{2}$ index $=0.10$, EGCG vs. tea drink; total between $\mathrm{Q}=3.835, p$-value $=0.050, \mathrm{R}^{2}$ index $=0.12$, EGCG vs. tea extract) and for TAGs (total between $\mathrm{Q}=3.282, p$-value $=0.070, \mathrm{R}^{2}$ index $=0.06$, EGCG vs. tea drink; total between $\mathrm{Q}=3.765, p$-value $=0.052, \mathrm{R}^{2}$ index $=0.09, \mathrm{EGCG}$ vs. tea extract). 
Table 4. Analysis of the influence of medication and health status on the effects (SDM) of the supplementation with flavanols on BMI, WC, and blood lipids levels.

\begin{tabular}{|c|c|c|c|c|c|c|c|c|c|}
\hline Factor & \multicolumn{2}{|c|}{ Medication } & \multicolumn{3}{|c|}{ Health Status } & \multicolumn{4}{|c|}{ Type of Disorder } \\
\hline Subgroup & Yes & No & $\begin{array}{c}\text { Healthy } \\
\text { Individuals }\end{array}$ & $\begin{array}{c}\text { Individuals } \\
\text { at Risk }\end{array}$ & $\begin{array}{c}\text { Individuals with } \\
\text { a Disease }\end{array}$ & Overweight/Obese & $\begin{array}{c}\text { Lipid } \\
\text { Disorders }\end{array}$ & $\begin{array}{c}\text { Glucose } \\
\text { Disorders }\end{array}$ & $\begin{array}{c}\text { Blood Pressure } \\
\text { Disorders }\end{array}$ \\
\hline $\begin{array}{c}\text { BMI } \\
p \text {-value } \\
(n, \mathrm{~N})\end{array}$ & $\begin{array}{c}-0.053^{\mathrm{a}} \\
\text { (NS) } \\
(15,744)\end{array}$ & $\begin{array}{c}-0.222 \\
(0.001) \\
(28,1312) \\
\end{array}$ & $\begin{array}{c}-0.119 \\
\text { (NS) } \\
(18,868)\end{array}$ & $\begin{array}{c}-0.195 \\
(0.009) \\
(26,1753) \\
\end{array}$ & $\begin{array}{c}-0.128 \\
(0.069) \\
(25,1008) \\
\end{array}$ & $\begin{array}{c}-0.191 \\
(0.011) \\
(22,1488) \\
\end{array}$ & $\begin{array}{c}-0.063 \\
(\mathrm{NS}) \\
(4,179) \\
\end{array}$ & $\begin{array}{c}-0.028 \\
(\mathrm{NS}) \\
(5,265)\end{array}$ & $\begin{array}{c}-0.054 \\
(\mathrm{NS}) \\
(5,225)\end{array}$ \\
\hline $\begin{array}{c}\text { WC } \\
p \text {-value } \\
(n, \mathrm{~N})\end{array}$ & $\begin{array}{c}-0.363 \\
(\mathrm{NS}) \\
(5,309)\end{array}$ & $\begin{array}{c}-0.445 \\
(0.003) \\
(21,1237)\end{array}$ & $\begin{array}{c}-0.092 \\
(\mathrm{NS}) \\
(4,250)\end{array}$ & $\begin{array}{c}-0.392 \\
(0.000) \\
(26,1485)\end{array}$ & $\begin{array}{c}-0.169 \\
(\mathrm{NS}) \\
(9,466)\end{array}$ & $\begin{array}{c}-0.426 \\
(0.000) \\
(25,1447)\end{array}$ & $\mathrm{NI}$ & $\begin{array}{c}-0.057 \\
(\mathrm{NS}) \\
(4,188)\end{array}$ & NI \\
\hline $\begin{array}{c}\text { TC } \\
p \text {-value } \\
(n, \mathrm{~N})\end{array}$ & $\begin{array}{c}-0.205 \\
(0.089) \\
(24,1174)\end{array}$ & $\begin{array}{c}-0.296 \\
(0.001) \\
(47,2169)\end{array}$ & $\begin{array}{c}-0.266 \\
(0.015) \\
(38,1520)\end{array}$ & $\begin{array}{c}-0.161 \\
(\mathrm{NS}) \\
(32,2243)\end{array}$ & $\begin{array}{c}-0.241 \\
(0.014) \\
(38,1859)\end{array}$ & $\begin{array}{c}-0.148 \\
\text { (NS) } \\
(24,1813)\end{array}$ & $\begin{array}{c}-0.227 \\
(\mathrm{NS}) \\
(9,491)\end{array}$ & $\begin{array}{c}-0.372 \\
(\mathrm{NS}) \\
(8,463)\end{array}$ & $\begin{array}{c}-0.515 \\
(\mathrm{NS}) \\
(5,275)\end{array}$ \\
\hline $\begin{array}{c}\text { LDL-C } \\
p \text {-value } \\
(n, \mathrm{~N})\end{array}$ & $\begin{array}{c}-0.200 \\
\text { (NS) } \\
(21,1020)\end{array}$ & $\begin{array}{c}-0.289 \\
(0.004) \\
(43,2311) \\
\end{array}$ & $\begin{array}{c}-0.210 \\
(0.028) \\
(37,1782) \\
\end{array}$ & $\begin{array}{c}-0.192 \\
(0.061) \\
(29,2049) \\
\end{array}$ & $\begin{array}{c}-0.311 \\
(0.003) \\
(36,1756) \\
\end{array}$ & $\begin{array}{c}-0.187 \\
(0.082) \\
(23,1742) \\
\end{array}$ & $\begin{array}{c}-0.336 \\
(\mathrm{NS}) \\
(9,491)\end{array}$ & $\begin{array}{l}-0.579 \\
(0.042) \\
(6,408)\end{array}$ & $\begin{array}{l}-0.754 \\
(0.041) \\
(4,146) \\
\end{array}$ \\
\hline $\begin{array}{c}\text { HDL-C } \\
p \text {-value } \\
(n, \mathrm{~N})\end{array}$ & $\begin{array}{c}0.162 \\
(\mathrm{NS}) \\
(25,1212)\end{array}$ & $\begin{array}{c}0.128 \\
(\mathrm{NS}) \\
(48,2042)\end{array}$ & $\begin{array}{c}0.163 \\
(\mathrm{NS}) \\
(37,1670)\end{array}$ & $\begin{array}{c}0.229 \\
-0.055 \\
(32,2187))\end{array}$ & $\begin{array}{c}0.091 \\
(\mathrm{NS}) \\
(39,1881)\end{array}$ & $\begin{array}{c}-0.062 \\
(\mathrm{NS}) \\
(20,1541)\end{array}$ & $\begin{array}{c}0.476 \\
-0.005 \\
(12,654)\end{array}$ & $\begin{array}{c}0.032 \\
(\mathrm{NS}) \\
(8,488)\end{array}$ & $\begin{array}{l}0.115 \\
(\mathrm{NS}) \\
(6,301)\end{array}$ \\
\hline $\begin{array}{c}\text { TAGs } \\
p \text {-value } \\
(n, \mathrm{~N})\end{array}$ & $\begin{array}{c}-0.165 \\
(\mathrm{NS}) \\
(23,1132)\end{array}$ & $\begin{array}{c}-0.135 \\
-0.063 \\
(63,3019)\end{array}$ & $\begin{array}{c}-0.144 \\
(\mathrm{NS}) \\
(45,2669)\end{array}$ & $\begin{array}{c}-0.08 \\
(\mathrm{NS}) \\
(31,1205)\end{array}$ & $\begin{array}{c}-0.103 \\
\text { (NS) } \\
(39,1931)\end{array}$ & $\begin{array}{c}-0.008 \\
\text { (NS) } \\
(24,912)\end{array}$ & $\begin{array}{c}-0.258 \\
(\mathrm{NS}) \\
(12,585)\end{array}$ & $\begin{array}{c}0.065 \\
(\mathrm{NS}) \\
(7,536)\end{array}$ & $\begin{array}{c}-0.251 \\
(\mathrm{NS}) \\
(6,250)\end{array}$ \\
\hline
\end{tabular}

a Standardized difference in means (SDM); BMI: Body Mass Index; WC: Waist Circumference; TC: Total Cholesterol; LDL-C: Low density Lipoprotein Cholesterol; HDL-C: High Density Lipoprotein Cholesterol; TAGs: Triacylglycerides; $p$-value $<0.05$ was considered significant ; $p$-value $<0.1$ and $\geq 0.05$ was considered marginally significant ; NS: No significant change/effect; $(n)$ : Number of studies included; $(\mathrm{N})$ : Total number of participants. 
Table 5. Analysis of the influence of the original source of flavanols and of the diet (during the intervention) on the effects (SDM) of the supplementation on BMI, WC, and blood lipids levels.

\begin{tabular}{|c|ccc|ccc|cc|}
\hline Factor & \multicolumn{7}{|c|}{ Source of Flavanols } & \multicolumn{2}{c|}{$\begin{array}{c}\text { Diet during } \\
\text { Supplementation }\end{array}$} \\
\hline Subgroup & $\begin{array}{c}\text { Cocoa } \\
\text { Products }\end{array}$ & $\begin{array}{c}\text { Apple } \\
\text { Products }\end{array}$ & $\begin{array}{c}\text { Tea } \\
\text { Products }\end{array}$ & $\begin{array}{c}\text { Tea } \\
\text { Drinks }\end{array}$ & $\begin{array}{c}\text { Tea } \\
\text { Extracts }\end{array}$ & $\begin{array}{c}\text { Tea Purified } \\
\text { EGCG }\end{array}$ & Controlled & Usual \\
\hline BMI & 0.001 a & -0.11 & -0.224 & -0.223 & -0.212 & -0.290 & -0.162 & -0.149 \\
$p$-value & $(\mathrm{NS})$ & $(\mathrm{NS})$ & $(0.000)$ & -0.007 & $(0.002)$ & $(0.022)$ & $(0.030)$ & $(0.001)$ \\
$(n, \mathrm{~N})$ & $(21,1014)$ & $(5,319)$ & $(46,2704)$ & $(20,1167)$ & $(26,1537)$ & $(7,384)$ & $(23,1032)$ & $(49,2990)$ \\
\hline WC & -0.106 & -0.206 & -0.354 & -0.22 & -0.506 & -0.465 & -0.500 & -0.164 \\
$p$-value & $(\mathrm{NS})$ & $(\mathrm{NS})$ & $(0.000)$ & $(\mathrm{NS})$ & $(0.000)$ & $(\mathrm{NS})$ & $(0.000)$ & $(0.084)$ \\
$(n, \mathrm{~N})$ & $(8,430)$ & $(2,155)$ & $(34,2228)$ & $(16,1074)$ & $(17,1110)$ & $(2,171)$ & $(18,903)$ & $(27,1868)$ \\
\hline TC & -0.177 & -1.352 & -0.143 & -0.083 & -0.208 & -0.051 & -0.215 & -0.213 \\
$p$-value & -0.018 & -0.041 & $(0.036)$ & $(\mathrm{NS})$ & $(0.008)$ & $(\mathrm{NS})$ & $(0.032)$ & $(0.003)$ \\
$(n, \mathrm{~N})$ & $(38,1625)$ & $(7,402)$ & $(65,3666)$ & $(36,2051)$ & $(26,1615)$ & $(7,437)$ & $(39,1682)$ & $(73,4130)$ \\
\hline LDL-C & -0.252 & -0.587 & -0.191 & -0.119 & -0.270 & -0.216 & -0.184 & -0.260 \\
$p$-value & -0.013 & -0.007 & $(0.008)$ & -0.045 & $(0.000)$ & $(\mathrm{NS})$ & $(0.062)$ & $(0.000)$ \\
$(n, \mathrm{~N})$ & $(35,1577)$ & $(8,386)$ & $(61,3723)$ & $(35,2235)$ & $(26,1488)$ & $(7,206)$ & $(38,1540)$ & $(67,4186)$ \\
\hline HDL-C & 0.16 & 0.197 & 0.150 & 0.17 & 0.13 & -0.291 & 0.245 & 0.104 \\
$p$-value & $(\mathrm{NS})$ & $(\mathrm{NS})$ & $(0.031)$ & -0.071 & $(\mathrm{NS})$ & $(\mathrm{NS})$ & $(0.008)$ & $(\mathrm{NS})$ \\
$(n, \mathrm{~N})$ & $(37,1603)$ & $(7,386)$ & $(66,3820)$ & $(38,2292)$ & $(27,1484)$ & $(7,437)$ & $(40,1759)$ & $(72,4169)$ \\
\hline TAGs & -0.183 & -0.173 & -0.050 & -0.047 & -0.053 & 0.314 & -0.139 & -0.105 \\
$p$-value & -0.047 & $(\mathrm{NS})$ & $(\mathrm{NS})$ & $(\mathrm{NS})$ & $(\mathrm{NS})$ & -0.077 & $(\mathrm{NS})$ & $(\mathrm{NS})$ \\
$(n, \mathrm{~N})$ & $(39,1691)$ & $(10,455)$ & $(69,3700)$ & $(38,2121)$ & $(30,1544)$ & $(7,437)$ & $(46,1939)$ & $(73,3922)$ \\
\hline
\end{tabular}

a Standardized difference in means (SDM); BMI: Body Mass Index; WC: Waist Circumference; TC: Total Cholesterol; LDL-C: Low density Lipoprotein Cholesterol; HDL-C: High Density Lipoprotein Cholesterol; TAGs: Triacylglycerides; $p$-value $<0.05$ was considered significant ; $p$-value $<0.1$ and $\geq 0.05$ was considered marginally significant ; NS: No significant change/effect; $(n)$ : Number of studies included; (N): Total number of participants; EGCG: Epigallocatechin gallate.

Regarding the type of diet (controlled vs. usual) during supplementation with the flavanol-containing products, the reducing effects on BMI, WC, total- and LDL-cholesterol remained significant or marginally significant in both subgroups. The levels of TAGs were also reduced although not significantly. We detected, however, a significant increase in the HDL-cholesterol levels only in the subgroup that followed a controlled diet (Table 5). Further, statistical comparison of the two subgroups highlighted a significant difference on the reduction of WC between them (total between $Q=4.761$, $p$-value $=0.029)$ and a $7 \%$ explanation of the between-groups variance by this factor $\left(R^{2}\right.$ index $\left.=0.07\right)$.

3.4.4. Multiple Meta-Regression Analysis of BMI Modulators of the Response to Flavanol-Containing Products Consumption

Multiple meta-regression analysis was performed (Table 6) to derive the independent effect of some of the covariates previously found to partially explain some of the between-groups variance for BMI, i.e., baseline BMI (64\%), country where the study was carried out (East-Asian vs. all other countries) $(7 \%)$, medication use (34\%) and source of flavanols (tea vs. cocoa products) $(29 \%)$.

Although sex also appeared to contribute greatly to the BMI between-groups variance $\left(R^{2}=1\right)$, it was not included in the multiple regression due to the limited number of studies clearly reporting sex and used in the analysis. The full model reached statistical significance, with a large proportion of variance $(94 \%)$ accounted for and a considerable number of studies included ( $n=40$ studies). Both medication and source of flavanols were significantly correlated with the reducing effect on BMI, once controlled the influence of the other predictor. In particular, higher effects of the flavanols-products on BMI were found in the absence of the medication and with the consumption of tea products. 
Table 6. Main results of the multiple random-effects meta-regression model for the contribution of the covariates, medication and source of flavanols, on BMI response (Standardized difference in means).

\begin{tabular}{|c|c|c|c|c|c|c|}
\hline Covariate & Coefficient & SE & 95\% Lower & 95\% Upper & $\mathbf{Z}$ & $p$-Value \\
\hline Intercept & -0.0679 & 0.0899 & -0.2440 & 0.1082 & -0.76 & 0.4500 \\
\hline Medication (No vs. Yes) & 0.2481 & 0.0995 & 0.0530 & 0.4431 & 2.49 & 0.0127 \\
\hline Source of flavanols (Cocoa vs. Tea) & -0.3278 & 0.1022 & -0.5282 & -0.1274 & -3.21 & 0.0013 \\
\hline \multicolumn{7}{|l|}{ Test of the Model } \\
\hline $\mathrm{Q}_{\mathrm{R}}$ & \multicolumn{6}{|c|}{15.64} \\
\hline$d f$ & \multicolumn{6}{|c|}{2} \\
\hline$p$-value & \multicolumn{6}{|c|}{0.0004} \\
\hline $\mathrm{R}^{2}$ & \multicolumn{6}{|c|}{0.94} \\
\hline Number of studies included & \multicolumn{6}{|c|}{40 ( $54 \%$ of the total studies used in the meta-analysis) } \\
\hline
\end{tabular}

SE: standard error for each regression coefficient; Z: statistic for testing the statistical significance of each predictor; $P$ : probability level; $\mathrm{Q}_{\mathrm{R}}$ : statistic for testing the statistical significance of the full meta-regression model; $d f$ : degrees of freedom; $\mathrm{R}^{2}$ : proportion of total between-studies variance explained by the model analog.

\section{Discussion}

The consumption of flavanols may contribute to improve cardiometabolic health via the moderation of a range of associated risk factors. Recent meta-analyses (Table S3) [138-151] suggest that the consumption of flavanol-containing tea and tea products could reduce total- and LDL-cholesterol as well as body mass index (BMI) and waist circumference (WC), while chocolate and cocoa flavanols also appear to regulate blood lipid levels. Nonetheless, the results of these analyses are inconsistent, partly due to the large heterogeneity of the clinical trials included. In addition, some of the anthropometric indicators of obesity such as BMI and WC have not yet been systematically investigated. We herein present the largest meta-analysis investigating the impact of flavanol-containing tea, cocoa and apple products, three major dietary sources of these bioactive compounds [152] on several biomarkers of lipid metabolism and anthropometric variables, such as BMI and WC. Our analysis confirms that the intake of these products is significantly associated with: (1) reduced BMI and WC; and (2) a more favorable lipid profile with a decrease in total- and LDL-cholesterol, and TAG plasma levels, and an increase in HDL-cholesterol levels. In addition, our analyses show that the changes in these biomarkers following consumption of the flavanol-containing products can be influenced by a number of factors and thus, the benefits of these products can significantly vary between specific population subgroups. It is of utmost interest to clarify the impact of these factors in order to discern which population subgroups could most benefit of the intake of these bioactive compounds.

\subsection{Baseline BMI}

There is evidence that baseline BMI may be a potential factor with an impact on the individuals' response to supplementation with different natural products. For instance, treatment with natural probiotics has been shown to significantly increase HDL only in patients with a baseline BMI $\geq 29 \mathrm{~kg} / \mathrm{m}^{2}$ [153] or significantly reduce BMI only in participants with a baseline BMI $\geq 25 \mathrm{~kg} / \mathrm{m}^{2}$ [154]. Regarding flavanol-containing products, a previous meta-analysis of the effects of black tea on blood cholesterol failed to detect differences in the modulation of cholesterol levels between individuals with normal weight or overweight and obese phenotype, but the results of this meta-analysis were estimated using a very small number of trials per subgroup (4 and 5 , respectively) [141]. Our stratification approach by baseline BMI provides some evidence that the changes following consumption of flavanol-containing products on BMI, WC and cholesterol levels are more pronounced in individuals with a baseline BMI $\geq 25 \mathrm{~kg} / \mathrm{m}^{2}$ and supports the fact that supplementation with these products may have a better impact on these risk factors in overweight and/or obese people. Nevertheless, it is not yet clear whether there is a general better efficacy of natural treatments in overweight and/or obese people, or if the effects may vary depending on the biomarkers or the products investigated. More trials in individuals with a normal $\mathrm{BMI}<25.0 \mathrm{~kg} / \mathrm{m}^{2}$ are still needed to further compare and demonstrate significant differences in the benefits of flavanol-containing 
products in relation to body weight, since most are conducted in populations of greater cardiometabolic risk, who are often obese in nature.

\subsection{Sex}

Understanding the differing responses by sex is becoming increasingly important. Previous work has shown that the reducing effects of green tea on total and LDL-cholesterol were significantly greater in men than in women, giving preliminary evidence of that supplementation with flavanol-containing green tea could have a different effect depending on the sex of the individuals [142]. Our results also support differences between women and men in their capacity to regulate the levels of total and LDL cholesterol in response to the consumption of flavanol-containing products with women exhibiting a more efficient reduction than men. A recent meta-analysis looking at the effects of flavonols (another flavonoids class) on lipids levels, failed to detect a difference between men and women, possibly due to the very low number of trials and participants in the two subgroups [7]. Comparing the regulation of cardiometabolic risk factors between women and men is complex because of the hormonal protection in premenopausal women [155]. We were not able to stratify our analyses based on the age or menopausal status of the women, as these factors were not sufficiently well characterized in the trials selected for the meta-analyses. Nevertheless, our results point out to a different response to flavanols consumption between sexes and reinforce the need to further investigate this factor in future trials specifically designed for this purpose.

\subsection{Country Where the Study Was Carried Out}

Ethno-cultural differences are associated with the risk of development of cardiometabolic disorders [156] and thus, it is important to explore and clarify whether different ethnic groups differ in their responses to consumption of plant bioactives as effective treatment against these diseases. Unfortunately, most of the clinical trials included in the present meta-analysis have not clearly identified the ethnicity of the participants. In the absence of this information, we have explored the potential influence of the country where the studies were carried out. A common comparison is that between studies undertaken in Asian countries vs. non-Asian ones. It has been reported that Asians showed a more marked decrease in the levels of TAGs in response to $\varpi-3$ fatty acids supplementation as compared to subjects within a USA/European group but, no significant differences were found for total cholesterol or BMI [157]. Flavonols have also been shown to significantly reduce TAGs, total and LDL-cholesterol in studies conducted in Asian countries as compared to those in the EU/European subgroup [7]. Regarding flavanol-containing products, previous meta-analyses have suggested that tea and tea extracts reduce BMI and WC both in Asian and non-Asian trials [140] and that, cocoa products significantly reduce LDL-cholesterol in European countries as compared to USA [149]. Nevertheless, these analyses were all underpowered. Our stratification analysis by country included, in general, a big number of studies per subgroup and showed no apparent differences in the responses to the consumption of tea, cocoa and apple products between East Asian countries and all other countries except for TAGs which were significantly reduced only in the Asian subgroup. We also found some different responses between North American (USA/Canada) and European subgroups, as well as between European Mediterranean and non-Mediterranean ones. This may be partially related to features such as the ethnicity of the participants but also to other factors associated with the life-style of the country. More studies are needed in order to understand the influence of this factor in the response to interventions with plant natural compounds.

\subsection{Health and Medication Status}

Previous meta-analyses had suggested that the consumption of green tea [146,147], black tea [139], and cocoa products [149] had moderating effects on lipid levels both in healthy subjects and in patients with hyperlipidemia or at a higher cross-over or cross-over or s risk. Other bioactive compounds such as flavonols also had a more pronounced effect in the disease subgroup than in the healthy subgroup 
as significantly evidenced for LDL-cholesterol [7]. Our results show and corroborate a significant reduction of total- and LDL-cholesterol by the flavanol-containing tea, cocoa and apple products both in healthy participants and in individuals with a disease. On the other hand, BMI and WC were reduced and HDL increased in the three subgroups of healthy, "at risk" and individuals with a disease but the results reached statistical significance in the "at risk" group only. As a whole, these results support a metabolic benefit of the consumption of plant bioactive compounds, and in particular of flavanols, regardless of the health status of the individuals.

An important consideration regarding the use of plant bioactive compounds as modulators of cardiometabolic risk biomarkers is their potential use as treatment on their own or as coadjuvants in combination with pharmacological drugs [158]. Our results show that the use of flavanol-containing products in the absence of medication was significantly associated with the reduction of BMI, WC, total and LDL cholesterol, as well as TAGs giving some evidence of their efficacy as therapeutics. The number of clinical trials in which the flavanols were supplemented in combination with other drugs was in general smaller than studies carried out in the absence of medication (see Table 4) thus the pooled results did not reach significance. Nonetheless, these data point to a modulatory effect of the flavanol-containing products in medicated individuals. Whether the combined therapy is more efficient and safe than individual treatment with drugs or with natural plant bioactives warrants further investigation.

\subsection{Source and Form of Administration of the Flavanols}

Our results confirm that the flavanol-containing tea products are effective regulators of blood cholesterol (total, LDL and HDL) as well as of BMI and WC. The cocoa or apple products were effective at reducing total- and LDL-cholesterol and the cocoa products were also able to significantly decrease the levels of TAGs. These results might suggest that the metabolic regulatory efficacy of these three flavanol-containing products could be ranked as tea > cocoa $>$ apple but caution should be taken with this interpretation due to the differences in the number of studies carried out with each source of flavanols as well as the differences in the doses and the composition of the products. Further studies are needed to corroborate this comparison. Our analysis also suggests that the administration of tea as a solid extract might be more efficient than tea beverages at reducing WC and total cholesterol. Earlier meta-analyses had suggested that the type of administration of green or black tea either in solid form (extracts and, capsules) or as a drink did not differ at reducing total- and LDL-cholesterol [139,146,147]. Unlike those previous analyses, where the number of studies per subgroup was very small, our stratification between tea drinks and tea extracts included a considerable number of studies per subgroup ( $>15)$ and gives preliminary evidence of a potentially higher efficacy of the tea when administered as a solid powder. We may hypothesize that this could be partially related to the presence of higher doses of the bioactive flavanols in such extracts.

\subsection{Magnitude of the Changes}

An interesting issue worth discussing here is the magnitude of the changes attributed to the intake of the flavanol-containing products and to the extent these changes can contribute to the regulation of the analyzed biomarkers in comparison with other approaches, i.e., drugs, lifestyle changes, or other natural compounds. Based on the Cohen guidelines [159], the effects of the flavanol-containing products (expressed as SDM) are, in general, small $(\leq 0.2)$ or medium (between 0.2 and 0.5 ) although changes in some specific risk markers in some specific subgroups can be considered high $(\geq 0.8)$. We used the same random effects model to generate the overall size effects by computing the difference in means (Table S4) and compare these values to some of the reported effects of pharmacological, behavioral or dietary interventions on BMI, WC and lipid levels. Some of the most potent reducing effects on BMI can be achieved with restricted energy $\operatorname{diet}\left(-2.7 \mathrm{~kg} / \mathrm{m}^{2}\right)$ [160], pharmacological interventions $\left(-1.3 \mathrm{~kg} / \mathrm{m}^{2}\right)$ [161] or behavioral (diet, exercise) interventions $(-0.9$ to $\left.-1.2 \mathrm{~kg} / \mathrm{m}^{2}\right)[162,163]$. These reductions constitute between $5 \%$ and $10 \%$ of the WHO established 
limit values for overweight $\left(B M I=25.0-29.9 \mathrm{~kg} / \mathrm{m}^{2}\right)$ and obesity $\left(B M I \geq 30.0 \mathrm{~kg} / \mathrm{m}^{2}\right)$. Alternatively, intervention with probiotics [154] or nutraceuticals (e.g., lipoic acid) [164] shows a more modest but also significant reduction of BMI (approximately $-0.5 \mathrm{~kg} / \mathrm{m}^{2}, \sim 2 \%$ change of the WHO values). On average, the size effect of the flavanol-containing products on BMI was smaller $\left(-0.15 \mathrm{~kg} / \mathrm{m}^{2}\right.$, Table S3) but, notably, this effect may be enhanced in specific subpopulations (up to $-0.91 \mathrm{~kg} / \mathrm{m}^{2}$ in studies conducted in European Mediterranean countries), more similar to other behavioral or dietary interventions. WC can also be significantly and efficiently reduced by brisk walking $(-2.83 \mathrm{~cm}, \sim 3 \%$ of the established 102/88 cm risk values) [162] and, more modestly $(-0.53 \mathrm{~cm}, \sim 0.5 \%$ of the established risk values) by intervention with supplements such as $₫-3$ polyunsaturated fatty acids [165]. Along these lines, intervention with the flavanol-containing products significantly reduces $W C$ by $1.7 \mathrm{~cm}$ and can reach reducing values of $-4.58 \mathrm{~cm}$ in studies conducted in European Mediterranean countries.

Regarding the cholesterol lowering effects, statins remain, at present, the first-choice agents. The pooled effects of various statins on total- and LDL-cholesterol were $-0.89 \mathrm{mmol} / \mathrm{L}(\sim 17 \%$ of the desirable $5.17 \mathrm{mmol} / \mathrm{L}$ limit level) and $-0.92 \mathrm{mmol} / \mathrm{L}(\sim 27 \%$ of the near optimal $3.36 \mathrm{mmol} / \mathrm{L}$ level), respectively [166]. Intervention with natural products such as red yeast rice or spirulina can be as effective as the statins, whereas other plant dietary bioactive compounds such as soluble fiber, sterols/stanols, probiotics and flavonols also significantly reduce total- and LDL-cholesterol by $0.5-0.1 \mathrm{mmol} / \mathrm{L}[7,167]$. In this context, the flavanol-containing products show a similar efficiency at lowering total cholesterol $(-0.13 \mathrm{mmol} / \mathrm{L})$ and LDL cholesterol $(-0.17 \mathrm{mmol} / \mathrm{L})$. Again, in specific subgroups (e.g., supplementation with flavanol-containing apple products) the reduction of total cholesterol was much more efficient $(-0.44 \mathrm{mmol} / \mathrm{L})$. These results are very relevant considering that the reduction of LDL-cholesterol by $1 \mathrm{mmol} / \mathrm{L}$ has been associated with a $23 \%$ reduction of CVDs risk [168] and reinforce the interest in understanding the influence of different factors on the regulatory efficiency of plant bioactive compounds, in general, and of flavanols in particular.

\subsection{Additional Recent Evidences}

Since the completion of this meta-analysis, additional RCTs investigating the effects of tea or cocoa products containing flavanols on lipid and anthropometric variables have been added to the existing literature [111,169-183]. The heterogeneity of these trials remains high with population samples including mixed sexes and ages, obese, overweight, healthy, hyperlipidemic and/or diabetic subjects, etc. The products were administered in different forms, mostly as green tea extracts/capsules or cocoa drinks and at different doses and intervention periods. Some of these studies further support the reduction of total- and LDL-cholesterol by green tea or cocoa flavanols or the increase of HDL by dark chocolate or cocoa [111,169-171]. Others show no significant effects on these variables [175]. Noteworthy, some of these trials included stratification analyses by baseline conditions, medication, disease, age, sex, or even genotype and further point to specific responses in some subgroups $[169,172,174,176,179]$. Likewise, the intake of a cocoa product caused a greater increased of HDL in normocholesterolemic patients than in dyslipidemic patients [176], green tea capsules caused a significant reduction of total-cholesterol in women with a cholesterol baseline value above $5.17 \mathrm{mmol} / \mathrm{L}$ [169] or of LDL-cholesterol in patients not receiving anti-hyperlipidemic drugs [179]. Of note, the interactions between two factors: baseline BMI and catechol-O-methyltransferase (COMT) genotype, was also recently investigated although the COMT genotype did not modify the effect of green tea extract on any of the variables investigated including BMI [172]. In our study, we were able to identify several factors that may contribute to explaining the heterogeneity on the BMI changes in response to the flavanol-containing products. By multiple meta-regression analysis, we also found that supplementation with these products may be most effective at reducing BMI when specifically using tea products in non-medicated patients. These results highlight the importance of understanding not only the factors affecting the variability in the responses but also the interactions between these factors. 


\section{Conclusions}

To the best of our knowledge, the meta-analysis conducted here is the largest one to date that compiles the evidence on the effects on various metabolic risk factors after supplementation with three sources of flavanols, tea, cocoa, and apple products. Our results show consistent and significant modulatory effects on BMI, WC and lipid levels. The size of these effects is modest but similar to that prompted by other natural products. We have also presented evidence of the influence of several factors on these beneficial effects that suggest that flavanols might be very effective in specific subpopulations such as overweight people or non-medicated individuals or when the source of these bioactive compounds is tea. Moreover, a combination of these factors may best explain interindividual variability in the response to the flavanols-containing products.

Although the total number of studies included in the meta-analysis was quite large, the number of studies (and of participants) remained small in some of the subgroup analyses. In addition, many of the studies reported limited or unclear information about the potential factors that may influence the treatment. These limitations affect the capability of the meta-analysis to unequivocally detect moderator variables and limit the significance of our findings. More randomized comparison studies with larger number of well-phenotyped volunteers and providing detailed descriptions of the participants and study characteristics are still needed. This research is crucial for a better understanding of the factors most relevantly involved in the variability of the responses to the consumption of these compounds and to achieve maximum efficacy so that flavanols may become an effective non-pharmacological alternative to battle hyperlipidemia, overweight/obesity and associated cardiometabolic disorders in humans.

Supplementary Materials: The following are available online at www.mdpi.com/2072-6643/9/7/746/s1. Figure S1: Forest plot of the meta-analysis evaluating the effects of supplementation with flavanols-containing tea, cocoa or apple products on human body mass index (BMI). A total of 74 studies (displayed in alphabetical order) were analysed. Pooled results are shown at the bottom using a random-effects model. SDM: Standardized difference in means, SE: standard error, 95\% CI: lower and upper confidence limits for the average SDM, RW: relative weight; Figure S2: Forest plot of the meta-analysis evaluating the effects of supplementation with flavanols-containing tea, cocoa or apple products on human waist circumference (WC). A total of 46 studies (displayed in alphabetical order) were analysed. Pooled results are shown at the bottom using a random-effects model. SDM: standardized difference in means, SE: standard error, 95\% CI: lower and upper confidence limits for the average SDM, RW: relative weight; Figure S3: Forest plot of the meta-analysis evaluating the effects of a prolonged supplementation with flavanols-containing tea, cocoa or apple products on human blood levels of total cholesterol. A total of 112 studies (displayed in alphabetical order) were analysed. Pooled results are shown at the bottom using a random-effects model. SDM: standardized difference in means, SE: standard error, 95\% CI: lower and upper confidence limits for the average SDM, RW: relative weight; Figure S4: Forest plot of the meta-analysis evaluating the effects of a prolonged supplementation with flavanols-containing tea, cocoa or apple products on human blood levels of LDL cholesterol. A total of 105 studies (displayed in alphabetical order) were analysed. Pooled results are shown at the bottom using a random-effects model. SDM: standardized difference in means, SE: standard error, 95\% CI: lower and upper confidence limits for the average SDM, RW: relative weight; Figure S5: Forest plot of the meta-analysis evaluating the effects of a prolonged supplementation with flavanols-containing tea, cocoa or apple products on human blood levels of HDL cholesterol. A total of 112 studies (displayed in alphabetical order) were analysed. Pooled results are shown at the bottom using a random-effects model. SDM: standardized difference in means, SE: standard error, 95\% CI: lower and upper confidence limits for the average SDM, RW: relative weight; Figure S6: Forest plot of the meta-analysis evaluating the effects of a prolonged supplementation with flavanols-containing tea, cocoa or apple products on human blood levels of triglycerides (TAGs). A total of 120 studies (displayed in alphabetical order) were analysed. Pooled results are shown at the bottom using a random-effects model. SDM: standardized difference in means, SE: standard error, 95\% CI: lower and upper confidence limits for the average SDM, RW: relative weight; Figure S7: Funnel plot and Eager statistics (intercept and 2-tailed $p$-value) of the meta-analysis evaluating the effects of a prolonged supplementation with flavanols-containing tea, cocoa or apple products on human BMI; Figure S8: Funnel plot and Eager statistics (intercept and 2-tailed $p$-value) of the meta-analysis evaluating the effects of a prolonged supplementation with flavanols-containing tea, cocoa or apple products on human WC; Figure S9: Funnel plot and Eager statistics (intercept and 2-tailed $p$-value) of the meta-analysis evaluating the effects of a prolonged supplementation with flavanols-containing tea, cocoa or apple products on human blood levels of total cholesterol; Figure S10: Funnel plot and Eager statistics (intercept and 2-tailed $p$-value) of the meta-analysis evaluating the effects of a prolonged supplementation with flavanols-containing tea, cocoa or apple products on human blood levels of LDL cholesterol; Figure S11: Funnel plot and Eager statistics (intercept and 2-tailed $p$-value) of the meta-analysis evaluating the effects of a prolonged supplementation with flavanols-containing tea, 
cocoa or apple products on human blood levels of HDL cholesterol; Figure S12: Funnel plot and Eager statistics (intercept and 2-tailed $p$-value) of the meta-analysis evaluating the effects of a prolonged supplementation with flavanols-containing tea, cocoa or apple products on human blood levels of triglycerides (TAGs); Table S1: Mean content (mg/100 fresh weight, FW) in flavonoids of green and black tea infusions, cocoa powder and whole apple illustrative of the composition of the three main sources of flavanols examined in this study: tea, cocoa and apple (data are based on the Phenol-Explorer database); Table S2: Results of the meta-regression of the changes in BMI, WC and blood lipids levels vs. duration of the supplementation with the flavanol-containing tea, cocoa or apple products; Table S3: Summary of most recent meta-analysis looking at the effects of flavanol-containing tea or cocoa products on anthropometric measurements and blood lipids associated with the development of metabolic disorders; Table S4: Overall effect size estimations (DM) for the impact of flavanols containing products on BMI, WC and blood lipids levels.

Acknowledgments: This article is based upon work from COST Action FA1403_POSITIVe "Interindividual variation in response to consumption of plant food bioactives and determinants involved" supported by COST (European Cooperation in Science and Technology, http://www.cost.eu/). The authors offer thanks for the financial support of the COST Action FA1403 "POSITIVe" to conduct two short-term scientific missions to A.G.-S. and P.P. at the University of Glasgow (E.C.) during which the data analysis was performed, and to M.G.-A. at the University College Dublin (E.G.) during which the protocol was developed. M.G.-A. also thanks to the Spanish Ministry of Economy and Competitiveness (MINECO) (PCIN-2014-133-MINECO, Spain), and CIBERFES (co-funded by the FEDER Program from EU).

Author Contributions: A.G.-S., A.R.-M., E.R.G., M.G.-A., E.C., P.P., C.M. and M.-T.G.-C. conceived and designed the study; A.G.-S., E.C., P.P., P.M., M.D'A., M.G.-A., A.R.-M., E.R.G., J.D., M.M., J.S.M., C.M., M.-T.G.-C. performed the data extraction; A.G.-S., J.S.M. and M.-T.G.-C. analyzed the data; A.G.-S., E.C., P.P., P.M., M.D'A., M.G.-A., A.R.-M., E.R.G., J.D., M.M., J.S.M., C.M., M.-T.G.-C. contributed to the discussions and preparation of the manuscript; and M.-T.G-C. wrote the article.

Conflicts of Interest: The authors declare no conflict of interest.

\section{References}

1. Pucci, G.; Alcidi, R.; Tap, L.; Battista, F.; Mattace-Raso, F.; Schillaci, G. Sex- and gender-related prevalence, cardiovascular risk and therapeutic approach in metabolic syndrome: A review of the literature. Pharmacol. Res. 2017, 120, 34-42. [CrossRef] [PubMed]

2. Nothlings, U.; Schulze, M.B.; Weikert, C.; Boeing, H.; van der Schouw, Y.T.; Bamia, C.; Benetou, V.; Lagiou, P.; Krogh, V.; Beulens, J.W.; et al. Intake of vegetables, legumes, and fruit, and risk for all-cause, cardiovascular, and cancer mortality in a european diabetic population. J. Nutr. 2008, 138, 775-781. [PubMed]

3. Howes, M.J.; Simmonds, M.S. The role of phytochemicals as micronutrients in health and disease. Curr. Opin. Clin. Nutr. Metab. Care 2014, 17, 558-566. [CrossRef] [PubMed]

4. Wang, S.; Moustaid-Moussa, N.; Chen, L.; Mo, H.; Shastri, A.; Su, R.; Bapat, P.; Kwun, I.; Shen, C.L. Novel insights of dietary polyphenols and obesity. J. Nutr. Biochem. 2014, 25, 1-18. [CrossRef] [PubMed]

5. Grosso, G.; Micek, A.; Godos, J.; Pajak, A.; Sciacca, S.; Galvano, F.; Giovannucci, E.L. Dietary flavonoid and lignan intake and mortality in prospective cohort studies: Systematic review and dose-response meta-analysis. Am. J. Epidemiol. 2017, 1-13. [CrossRef] [PubMed]

6. Rienks, J.; Barbaresko, J.; Nothlings, U. Association of polyphenol biomarkers with cardiovascular disease and mortality risk: A systematic review and meta-analysis of observational studies. Nutrients 2017, 9, 415. [CrossRef]

7. Menezes, R.; Rodriguez-Mateos, A.; Kaltsatou, A.; Gonzalez-Sarrias, A.; Greyling, A.; Giannaki, C.; Andres-Lacueva, C.; Milenkovic, D.; Gibney, E.R.; Dumont, J.; et al. Impact of flavonols on cardiometabolic biomarkers: A meta-analysis of randomized controlled human trials to explore the role of inter-individual variability. Nutrients 2017, 9, 117. [CrossRef] [PubMed]

8. Del Rio, D.; Rodriguez-Mateos, A.; Spencer, J.P.; Tognolini, M.; Borges, G.; Crozier, A. Dietary (poly)phenolics in human health: Structures, bioavailability, and evidence of protective effects against chronic diseases. Antioxid. Redox Signal. 2013, 18, 1818-1892. [CrossRef] [PubMed]

9. Rodriguez-Mateos, A.; Vauzour, D.; Krueger, C.G.; Shanmuganayagam, D.; Reed, J.; Calani, L.; Mena, P.; Del Rio, D.; Crozier, A. Bioavailability, bioactivity and impact on health of dietary flavonoids and related compounds: An update. Arch. Toxicol. 2014, 88, 1803-1853. [CrossRef] [PubMed]

10. Neveu, V.; Perez-Jiménez, J.; Vos, F.; Crespy, V.; du Chaffaut, L.; Mennen, L.; Knox, C.; Eisner, R.; Cruz, J.; Wishart, D.; et al. Phenol-explorer: An online comprehensive database on polyphenol contents in foods. Database 2010. [CrossRef] [PubMed] 
11. Vogiatzoglou, A.; Mulligan, A.A.; Luben, R.N.; Lentjes, M.A.; Heiss, C.; Kelm, M.; Merx, M.W.; Spencer, J.P.; Schroeter, H.; Kuhnle, G.G. Assessment of the dietary intake of total flavan-3-ols, monomeric flavan-3-ols, proanthocyanidins and theaflavins in the european union. Br. J. Nutr. 2014, 111, 1463-1473. [CrossRef] [PubMed]

12. Actis-Goretta, L.; Leveques, A.; Giuffrida, F.; Romanov-Michailidis, F.; Viton, F.; Barron, D.; Duenas-Paton, M.; Gonzalez-Manzano, S.; Santos-Buelga, C.; Williamson, G.; et al. Elucidation of (-)-epicatechin metabolites after ingestion of chocolate by healthy humans. Free Radic. Biol. Med. 2012, 53, 787-795. [CrossRef] [PubMed]

13. Manach, C.; Williamson, G.; Morand, C.; Scalbert, A.; Remesy, C. Bioavailability and bioefficacy of polyphenols in humans. I. Review of 97 bioavailability studies. Am. J. Clin. Nutr. 2005, 81, 230S-242S. [PubMed]

14. Ottaviani, J.I.; Kwik-Uribe, C.; Keen, C.L.; Schroeter, H. Intake of dietary procyanidins does not contribute to the pool of circulating flavanols in humans. Am. J. Clin. Nutr. 2012, 95, 851-858. [CrossRef] [PubMed]

15. Manach, C.; Milenkovic, D.; Van de Wiele, T.; Rodriguez-Mateos, A.; de Roos, B.; Garcia-Conesa, M.T.; Landberg, R.; Gibney, E.R.; Heinonen, M.; Tomas-Barberan, F.; et al. Addressing the inter-individual variation in response to consumption of plant food bioactives: Towards a better understanding of their role in healthy aging and cardiometabolic risk reduction. Mol. Nutr. Food Res. 2017. [CrossRef] [PubMed]

16. Liberati, A.; Altman, D.G.; Tetzlaff, J.; Mulrow, C.; Gotzsche, P.C.; Ioannidis, J.P.; Clarke, M.; Devereaux, P.J.; Kleijnen, J.; Moher, D. The prisma statement for reporting systematic reviews and meta-analyses of studies that evaluate health care interventions: Explanation and elaboration. PLoS Med. 2009, 6, e1000100. [CrossRef] [PubMed]

17. Higgins, J.P.T.; Green, S. (Eds.) Cochrane Handbook for Systematic Reviews of Interventions Version 5.1.0 [Updated March 2011]. The Cochrane Collaboration, 2011. Available online: http://handbook-5-1.cochrane. org/ (accessed on 15 May 2015).

18. Centre for Reviews and Dissemination (CRD). Systematic Reviews: CRD's Guidance for Undertaking Reviews in Health Care; CRD, University of York: York, UK, 2009.

19. Higgins, J.P.T.; Altman, D.G.; Sterne, J.A.C. (Eds.) Assessing Risk of Bias in Included Studies. In Cochrane Handbook for Systematic Reviews of Interventions Version 5.1.0 [Updated March 2011]. The Cochrane Collaboration, 2011. Available online: http:/ / handbook-5-1.cochrane.org/ (accessed on 15 October 2015).

20. Borenstein, M.J.; Hedges, L.V.; Higgins, J.; Rothstein, H. Comprehensive Meta-Analysis Vers. 3.3 [Computer Program]; Biostat, Inc.: Englewood, NJ, USA, 2014.

21. Akazome, Y.; Kametani, N.; Kanda, T.; Shimasaki, H.; Kobayashi, S. Evaluation of safety of excessive intake and efficacy of long-term intake of beverages containing apple polyphenols. J. Oleo Sci. 2010, 59, 321-338. [CrossRef] [PubMed]

22. Al-Faris, N.A. Short-term consumption of a dark chocolate containing flavanols is followed by a significant decrease in normotensive population. Pak. J. Nutr. 2008, 7, 773-781. [CrossRef]

23. Auvichayapat, P.; Prapochanung, M.; Tunkamnerdthai, O.; Sripanidkulchai, B.O.; Auvichayapat, N.; Thinkhamrop, B.; Kunhasura, S.; Wongpratoom, S.; Sinawat, S.; Hongprapas, P. Effectiveness of green tea on weight reduction in obese thais: A randomized, controlled trial. Physiol. Behav. 2008, 93, 486-491. [CrossRef] [PubMed]

24. Baba, S.; Natsume, M.; Yasuda, A.; Nakamura, Y.; Tamura, T.; Osakabe, N.; Kanegae, M.; Kondo, K. Plasma LDL and HDL cholesterol and oxidized LDL concentrations are altered in normo- and hypercholesterolemic humans after intake of different levels of cocoa powder. J. Nutr. 2007, 137, 1436-1441. [PubMed]

25. Baba, S.; Osakabe, N.; Kato, Y.; Natsume, M.; Yasuda, A.; Kido, T.; Fukuda, K.; Muto, Y.; Kondo, K. Continuous intake of polyphenolic compounds containing cocoa powder reduces LDL oxidative susceptibility and has beneficial effects on plasma HDL-cholesterol concentrations in humans. Am. J. Clin. Nutr. 2007, 85, 709-717. [PubMed]

26. Bahorun, T.; Luximon-Ramma, A.; Neergheen-Bhujun, V.S.; Gunness, T.K.; Googoolye, K.; Auger, C.; Crozier, A.; Aruoma, O.I. The effect of black tea on risk factors of cardiovascular disease in a normal population. Prev. Med. 2012, 54, S98-S102. [CrossRef] [PubMed]

27. Bajerska, J.; Mildner-Szkudlarz, S.; Walkowiak, J. Effects of rye bread enriched with green tea extract on weight maintenance and the characteristics of metabolic syndrome following weight loss: A pilot study. J. Med. Food 2015, 18, 698-705. [CrossRef] [PubMed] 
28. Balzer, J.; Rassaf, T.; Heiss, C.; Kleinbongard, P.; Lauer, T.; Merx, M.; Heussen, N.; Gross, H.B.; Keen, C.L.; Schroeter, H.; et al. Sustained benefits in vascular function through flavanol-containing cocoa in medicated diabetic patients a double-masked, randomized, controlled trial. J. Am. Coll. Cardiol. 2008, 51, 2141-2149. [CrossRef] [PubMed]

29. Barth, S.W.; Koch, T.C.; Watzl, B.; Dietrich, H.; Will, F.; Bub, A. Moderate effects of apple juice consumption on obesity-related markers in obese men: Impact of diet-gene interaction on body fat content. Eur. J. Nutr. 2012, 51, 841-850. [CrossRef] [PubMed]

30. Basu, A.; Du, M.; Sanchez, K.; Leyva, M.J.; Betts, N.M.; Blevins, S.; Wu, M.; Aston, C.E.; Lyons, T.J. Green tea minimally affects biomarkers of inflammation in obese subjects with metabolic syndrome. Nutrition 2011, 27, 206-213. [CrossRef] [PubMed]

31. Basu, A.; Sanchez, K.; Leyva, M.J.; Wu, M.; Betts, N.M.; Aston, C.E.; Lyons, T.J. Green tea supplementation affects body weight, lipids, and lipid peroxidation in obese subjects with metabolic syndrome. J. Am. Coll. Nutr. 2010, 29, 31-40. [CrossRef] [PubMed]

32. Batista Gde, A.; Cunha, C.L.; Scartezini, M.; von der Heyde, R.; Bitencourt, M.G.; Melo, S.F. Prospective double-blind crossover study of camellia sinensis (green tea) in dyslipidemias. Arq. Bras. Cardiol. 2009, 93, 128-134. [PubMed]

33. Belcaro, G.; Ledda, A.; Hu, S.; Cesarone, M.R.; Feragalli, B.; Dugall, M. Greenselect phytosome for borderline metabolic syndrome. Evid. Based Complement. Altern. Med. 2013, 2013, 869061. [CrossRef] [PubMed]

34. Bingham, S.A.; Vorster, H.; Jerling, J.C.; Magee, E.; Mulligan, A.; Runswick, S.A.; Cummings, J.H. Effect of black tea drinking on blood lipids, blood pressure and aspects of bowel habit. Br. J. Nutr. 1997, 78, 41-55. [CrossRef] [PubMed]

35. Bogdanski, P.; Suliburska, J.; Szulinska, M.; Stepien, M.; Pupek-Musialik, D.; Jablecka, A. Green tea extract reduces blood pressure, inflammatory biomarkers, and oxidative stress and improves parameters associated with insulin resistance in obese, hypertensive patients. Nutr. Res. 2012, 32, 421-427. [CrossRef] [PubMed]

36. Bohn, S.K.; Croft, K.D.; Burrows, S.; Puddey, I.B.; Mulder, T.P.; Fuchs, D.; Woodman, R.J.; Hodgson, J.M. Effects of black tea on body composition and metabolic outcomes related to cardiovascular disease risk: A randomized controlled trial. Food Funct. 2014, 5, 1613-1620. [CrossRef] [PubMed]

37. Bondia-Pons, I.; Poho, P.; Bozzetto, L.; Vetrani, C.; Patti, L.; Aura, A.M.; Annuzzi, G.; Hyotylainen, T.; Rivellese, A.A.; Oresic, M. Isoenergetic diets differing in their $n-3$ fatty acid and polyphenol content reflect different plasma and HDL-fraction lipidomic profiles in subjects at high cardiovascular risk. Mol. Nutr. Food Res. 2014, 58, 1873-1882. [CrossRef] [PubMed]

38. Brown, A.L.; Lane, J.; Coverly, J.; Stocks, J.; Jackson, S.; Stephen, A.; Bluck, L.; Coward, A.; Hendrickx, H. Effects of dietary supplementation with the green tea polyphenol epigallocatechin-3-gallate on insulin resistance and associated metabolic risk factors: Randomized controlled trial. Br. J. Nutr. 2009, 101, 886-894. [CrossRef] [PubMed]

39. Brown, A.L.; Lane, J.; Holyoak, C.; Nicol, B.; Mayes, A.E.; Dadd, T. Health effects of green tea catechins in overweight and obese men: A randomised controlled cross-over trial. Br. J. Nutr. 2011, 106, 1880-1889. [CrossRef] [PubMed]

40. Cardoso, G.A.; Salgado, J.M.; Cesar, M.D.C.; Donado-Pestana, C.M. The effects of green tea consumption and resistance training on body composition and resting metabolic rate in overweight or obese women. J. Med. Food. 2013, 16, 120-127. [CrossRef] [PubMed]

41. Chai, S.C.; Hooshmand, S.; Saadat, R.L.; Payton, M.E.; Brummel-Smith, K.; Arjmandi, B.H. Daily apple versus dried plum: Impact on cardiovascular disease risk factors in postmenopausal women. J. Acad. Nutr. Diet. 2012, 112, 1158-1168. [CrossRef] [PubMed]

42. Chan, E.K.; Quach, J.; Mensah, F.K.; Sung, V.; Cheung, M.; Wake, M. Dark chocolate for children's blood pressure: Randomised trial. Arch. Dis. Child. 2012, 97, 637-640. [CrossRef] [PubMed]

43. Chen, I.J.; Liu, C.Y.; Chiu, J.P.; Hsu, C.H. Therapeutic effect of high-dose green tea extract on weight reduction: A randomized, double-blind, placebo-controlled clinical trial. Clin. Nutr. 2016, 35, 592-599. [CrossRef] [PubMed]

44. Coimbra, S.; Santos-Silva, A.; Rocha-Pereira, P.; Rocha, S.; Castro, E. Green tea consumption improves plasma lipid profiles in adults. Nutr. Res. 2006, 26, 604-607. [CrossRef] 
45. Crew, K.D.; Ho, K.A.; Brown, P.; Greenlee, H.; Bevers, T.B.; Arun, B.; Sneige, N.; Hudis, C.; McArthur, H.L.; Chang, J.; et al. Effects of a green tea extract, polyphenon e, on systemic biomarkers of growth factor signalling in women with hormone receptor-negative breast cancer. J. Hum. Nutr. Diet. 2015, 28, 272-282. [CrossRef] [PubMed]

46. Crews, W.D., Jr.; Harrison, D.W.; Wright, J.W. A double-blind, placebo-controlled, randomized trial of the effects of dark chocolate and cocoa on variables associated with neuropsychological functioning and cardiovascular health: Clinical findings from a sample of healthy, cognitively intact older adults. Am. J. Clin. Nutr. 2008, 87, 872-880. [PubMed]

47. Davies, M.J.; Judd, J.T.; Baer, D.J.; Clevidence, B.A.; Paul, D.R.; Edwards, A.J.; Wiseman, S.A.; Muesing, R.A.; Chen, S.C. Black tea consumption reduces total and LDL cholesterol in mildly hypercholesterolemic adults. J. Nutr. 2003, 133, 3298S-3302S. [PubMed]

48. Davison, K.; Coates, A.M.; Buckley, J.D.; Howe, P.R. Effect of cocoa flavanols and exercise on cardiometabolic risk factors in overweight and obese subjects. Int. J. Obes. 2008, 32, 1289-1296. [CrossRef] [PubMed]

49. Romero-Prado, M.M.J.; Curiel-Beltran, J.A.; Miramontes-Espino, M.V.; Cardona-Munoz, E.G.; Rios-Arellano, A.; Balam-Salazar, L.B. Dietary flavonoids added to pharmacological antihypertensive therapy are effective in improving blood pressure. Basic Clin. Pharmacol. Toxicol. 2015, 117, 57-64. [CrossRef] [PubMed]

50. De la Torre, R.; De Sola, S.; Pons, M.; Duchon, A.; de Lagran, M.M.; Farre, M.; Fito, M.; Benejam, B.; Langohr, K.; Rodriguez, J.; et al. Epigallocatechin-3-gallate, a dyrk1a inhibitor, rescues cognitive deficits in down syndrome mouse models and in humans. Mol. Nutr. Food Res. 2014, 58, 278-288. [CrossRef] [PubMed]

51. Desideri, G.; Kwik-Uribe, C.; Grassi, D.; Necozione, S.; Ghiadoni, L.; Mastroiacovo, D.; Raffaele, A.; Ferri, L.; Bocale, R.; Lechiara, M.C.; et al. Benefits in cognitive function, blood pressure, and insulin resistance through cocoa flavanol consumption in elderly subjects with mild cognitive impairment: The cocoa, cognition, and aging (cocoa) study. Hypertension 2012, 60, 794-801. [CrossRef] [PubMed]

52. Diepvens, K.; Kovacs, E.M.; Vogels, N.; Westerterp-Plantenga, M.S. Metabolic effects of green tea and of phases of weight loss. Physiol. Behav. 2006, 87, 185-191. [CrossRef] [PubMed]

53. Dower, J.I.; Geleijnse, J.M.; Gijsbers, L.; Zock, P.L.; Kromhout, D.; Hollman, P.C. Effects of the pure flavonoids epicatechin and quercetin on vascular function and cardiometabolic health: A randomized, double-blind, placebo-controlled, crossover trial. Am. J. Clin. Nutr. 2015, 101, 914-921. [CrossRef] [PubMed]

54. Duffy, S.J.; Keaney, J.F., Jr.; Holbrook, M.; Gokce, N.; Swerdloff, P.L.; Frei, B.; Vita, J.A. Short- and long-term black tea consumption reverses endothelial dysfunction in patients with coronary artery disease. Circulation 2001, 104, 151-156. [CrossRef] [PubMed]

55. Erba, D.; Riso, P.; Bordoni, A.; Foti, P.; Biagi, P.L.; Testolin, G. Effectiveness of moderate green tea consumption on antioxidative status and plasma lipid profile in humans. J. Nutr. Biochem. 2005, 16, 144-149. [CrossRef] [PubMed]

56. Esser, D.; Mars, M.; Oosterink, E.; Stalmach, A.; Muller, M.; Afman, L.A. Dark chocolate consumption improves leukocyte adhesion factors and vascular function in overweight men. FASEB J. 2014, 28, 1464-1473. [CrossRef] [PubMed]

57. Farouque, H.M.; Leung, M.; Hope, S.A.; Baldi, M.; Schechter, C.; Cameron, J.D.; Meredith, I.T. Acute and chronic effects of flavanol-rich cocoa on vascular function in subjects with coronary artery disease: A randomized double-blind placebo-controlled study. Clin. Sci. 2006, 111, 71-80. [CrossRef] [PubMed]

58. Flammer, A.J.; Sudano, I.; Wolfrum, M.; Thomas, R.; Enseleit, F.; Periat, D.; Kaiser, P.; Hirt, A.; Hermann, M.; Serafini, M.; et al. Cardiovascular effects of flavanol-rich chocolate in patients with heart failure. Eur. Heart J. 2012, 33, 2172-2180. [CrossRef] [PubMed]

59. Frank, J.; George, T.W.; Lodge, J.K.; Rodriguez-Mateos, A.M.; Spencer, J.P.; Minihane, A.M.; Rimbach, G. Daily consumption of an aqueous green tea extract supplement does not impair liver function or alter cardiovascular disease risk biomarkers in healthy men. J. Nutr. 2009, 139, 58-62. [CrossRef] [PubMed]

60. Freese, R.; Basu, S.; Hietanen, E.; Nair, J.; Nakachi, K.; Bartsch, H.; Mutanen, M. Green tea extract decreases plasma malondialdehyde concentration but does not affect other indicators of oxidative stress, nitric oxide production, or hemostatic factors during a high-linoleic acid diet in healthy females. Eur. J. Nutr. 1999, 38, 149-157. [CrossRef] [PubMed] 
61. Fu, D.; Ryan, E.P.; Huang, J.; Liu, Z.; Weir, T.L.; Snook, R.L.; Ryan, T.P. Fermented camellia sinensis, fu zhuan tea, regulates hyperlipidemia and transcription factors involved in lipid catabolism. Food Res. Int. 2011, 44, 2999-3005. [CrossRef]

62. Fujita, H.; Yamagami, T. Antihypercholesterolemic effect of Chinese black tea extract in human subjects with borderline hypercholesterolemia. Nutr. Res. 2008, 28, 450-456. [CrossRef] [PubMed]

63. Fukino, Y.; Ikeda, A.; Maruyama, K.; Aoki, N.; Okubo, T.; Iso, H. Randomized controlled trial for an effect of green tea-extract powder supplementation on glucose abnormalities. Eur. J. Clin. Nutr. 2008, 62, 953-960. [CrossRef] [PubMed]

64. Grassi, D.; Lippi, C.; Necozione, S.; Desideri, G.; Ferri, C. Short-term administration of dark chocolate is followed by a significant increase in insulin sensitivity and a decrease in blood pressure in healthy persons. Am. J. Clin. Nutr. 2005, 81, 611-614. [PubMed]

65. Grassi, D.; Necozione, S.; Lippi, C.; Croce, G.; Valeri, L.; Pasqualetti, P.; Desideri, G.; Blumberg, J.B.; Ferri, C. Cocoa reduces blood pressure and insulin resistance and improves endothelium-dependent vasodilation in hypertensives. Hypertension 2005, 46, 398-405. [CrossRef] [PubMed]

66. Hodgson, J.M.; Croft, K.D.; Woodman, R.J.; Puddey, I.B.; Fuchs, D.; Draijer, R.; Lukoshkova, E.; Head, G.A. Black tea lowers the rate of blood pressure variation: A randomized controlled trial. Am. J. Clin. Nutr. 2013, 97, 943-950. [CrossRef] [PubMed]

67. Hodgson, J.M.; Puddey, I.B.; Burke, V.; Watts, G.F.; Beilin, L.J. Regular ingestion of black tea improves brachial artery vasodilator function. Clin. Sci. 2002, 102, 195-201. [CrossRef] [PubMed]

68. Hsu, C.H.; Liao, Y.L.; Lin, S.C.; Tsai, T.H.; Huang, C.J.; Chou, P. Does supplementation with green tea extract improve insulin resistance in obese type 2 diabetics? A randomized, double-blind, and placebo-controlled clinical trial. Altern. Med. Rev. 2011, 16, 157-163. [CrossRef] [PubMed]

69. Hsu, C.H.; Tsai, T.H.; Kao, Y.H.; Hwang, K.C.; Tseng, T.Y.; Chou, P. Effect of green tea extract on obese women: A randomized, double-blind, placebo-controlled clinical trial. Clin. Nutr. 2008, 27, 363-370. [CrossRef] [PubMed]

70. Hsu, T.F.; Kusumoto, A.; Abe, K.; Hosoda, K.; Kiso, Y.; Wang, M.F.; Yamamoto, S. Polyphenol-enriched oolong tea increases fecal lipid excretion. Eur. J. Clin. Nutr. 2006, 60, 1330-1336. [CrossRef] [PubMed]

71. Hursel, R.; Westerterp-Plantenga, M.S. Green tea catechin plus caffeine supplementation to a high-protein diet has no additional effect on body weight maintenance after weight loss. Am. J. Clin. Nutr. 2009, 89, 822-830. [CrossRef] [PubMed]

72. Ibero-Baraibar, I.; Abete, I.; Navas-Carretero, S.; Massis-Zaid, A.; Martinez, J.A.; Zulet, M.A. Oxidised LDL levels decreases after the consumption of ready-to-eat meals supplemented with cocoa extract within a hypocaloric diet. Nutr. Metab. Cardiovasc. Dis. 2014, 24, 416-422. [CrossRef] [PubMed]

73. Ichinose, T.; Nomura, S.; Someya, Y.; Akimoto, S.; Tachiyashiki, K.; Imaizumi, K. Effect of endurance training supplemented with green tea extract on substrate metabolism during exercise in humans. Scand. J. Med. Sci. Sports 2011, 21, 598-605. [CrossRef] [PubMed]

74. Inami, S.; Takano, M.; Yamamoto, M.; Murakami, D.; Tajika, K.; Yodogawa, K.; Yokoyama, S.; Ohno, N.; Ohba, T.; Sano, J.; et al. Tea catechin consumption reduces circulating oxidized low-density lipoprotein. Int. Heart J. 2007, 48, 725-732. [CrossRef] [PubMed]

75. Ishikawa, T.; Suzukawa, M.; Ito, T.; Yoshida, H.; Ayaori, M.; Nishiwaki, M.; Yonemura, A.; Hara, Y.; Nakamura, H. Effect of tea flavonoid supplementation on the susceptibility of low-density lipoprotein to oxidative modification. Am. J. Clin. Nutr. 1997, 66, 261-266. [PubMed]

76. Kajimoto, O.; Kajimoto, Y.; Yabune, M.; Nakamura, T.; Kotani, K.; Suzuki, Y.; Nozawa, A.; Nagata, K.; Unno, T.; Sagesaka, Y.M.; et al. Tea catechins with a galloyl moiety reduce body weight and fat. J. Health Sci. 2005, 51, 161-171. [CrossRef]

77. Kajimoto, O.; Kajimoto, Y.; Yabune, M.; Nozawa, A.; Nagata, K.; Kakuda, T. Tea catechins reduce serum cholesterol levels in mild and borderline hypercholesterolemia patients. J. Clin. Biochem. Nutr. 2003, 33, 101-111. [CrossRef]

78. Koutelidakis, A.E.; Rallidis, L.; Koniari, K.; Panagiotakos, D.; Komaitis, M.; Zampelas, A.; Anastasiou-Nana, M.; Kapsokefalou, M. Effect of green tea on postprandial antioxidant capacity, serum lipids, c-reactive protein and glucose levels in patients with coronary artery disease. Eur. J. Nutr. 2014, 53, 479-486. [CrossRef] [PubMed] 
79. Kovacs, E.M.; Lejeune, M.P.; Nijs, I.; Westerterp-Plantenga, M.S. Effects of green tea on weight maintenance after body-weight loss. Br. J. Nutr. 2004, 91, 431-437. [CrossRef] [PubMed]

80. Lettieri-Barbato, D.; Villaño, D.; Beheydt, B.; Guadagni, F.; Trogh, I.; Serafini, M. Effect of ingestion of dark chocolates with similar lipid composition and different cocoa content on antioxidant and lipid status in healthy humans. Food Chem. 2012, 132, 1305-1310. [CrossRef]

81. Liu, C.Y.; Huang, C.J.; Huang, L.H.; Chen, I.J.; Chiu, J.P.; Hsu, C.H. Effects of green tea extract on insulin resistance and glucagon-like peptide 1 in patients with type 2 diabetes and lipid abnormalities: A randomized, double-blinded, and placebo-controlled trial. PLoS ONE 2014, 9, e91163. [CrossRef] [PubMed]

82. Mahler, A.; Steiniger, J.; Bock, M.; Klug, L.; Parreidt, N.; Lorenz, M.; Zimmermann, B.F.; Krannich, A.; Paul, F.; Boschmann, M. Metabolic response to epigallocatechin-3-gallate in relapsing-remitting multiple sclerosis: A randomized clinical trial. Am. J. Clin. Nutr. 2015, 101, 487-495. [CrossRef] [PubMed]

83. Maki, K.C.; Reeves, M.S.; Farmer, M.; Yasunaga, K.; Matsuo, N.; Katsuragi, Y.; Komikado, M.; Tokimitsu, I.; Wilder, D.; Jones, F.; et al. Green tea catechin consumption enhances exercise-induced abdominal fat loss in overweight and obese adults. J. Nutr. 2009, 139, 264-270. [CrossRef] [PubMed]

84. Mastroiacovo, D.; Kwik-Uribe, C.; Grassi, D.; Necozione, S.; Raffaele, A.; Pistacchio, L.; Righetti, R.; Bocale, R.; Lechiara, M.C.; Marini, C.; et al. Cocoa flavanol consumption improves cognitive function, blood pressure control, and metabolic profile in elderly subjects: The cocoa, cognition, and aging (cocoa) study-A randomized controlled trial. Am. J. Clin. Nutr. 2015, 101, 538-548. [CrossRef] [PubMed]

85. Matsuyama, T.; Tanaka, Y.; Kamimaki, I.; Nagao, T.; Tokimitsu, I. Catechin safely improved higher levels of fatness, blood pressure, and cholesterol in children. Obes. Silver Spring 2008, 16, 1338-1348. [CrossRef] [PubMed]

86. Mellor, D.D.; Sathyapalan, T.; Kilpatrick, E.S.; Beckett, S.; Atkin, S.L. High-cocoa polyphenol-rich chocolate improves HDL cholesterol in type 2 diabetes patients. Diabet. Med. 2010, 27, 1318-1321. [CrossRef] [PubMed]

87. Mielgo-Ayuso, J.; Barrenechea, L.; Alcorta, P.; Larrarte, E.; Margareto, J.; Labayen, I. Effects of dietary supplementation with epigallocatechin-3-gallate on weight loss, energy homeostasis, cardiometabolic risk factors and liver function in obese women: Randomised, double-blind, placebo-controlled clinical trial. Br. J. Nutr. 2014, 111, 1263-1271. [CrossRef] [PubMed]

88. Mirzaei, K.; Hossein-Nezhad, A.; Karimi, M.; Hosseinzadeh-Attar, M.J.; Jafari, N.; Najmafshar, A.; Larijani, B. Effect of green tea extract on bone turnover markers in type 2 diabetic patients; a doubleblind, placebo-controlled clinical trial study. DARU J. Pharm. Sci. 2009, 17, 38-44.

89. Miyazaki, R.; Kotani, K.; Ayabe, M.; Tsuzaki, K.; Shimada, J.; Sakane, N.; Takase, H.; Ichikawa, H.; Yonei, Y.; Ishii, K. Minor effects of green tea catechin supplementation on cardiovascular risk markers in active older people: A randomized controlled trial. Geriatr. Gerontol. Int. 2013, 13, 622-629. [CrossRef] [PubMed]

90. Mogollon, J.A.; Bujold, E.; Lemieux, S.; Bourdages, M.; Blanchet, C.; Bazinet, L.; Couillard, C.; Noel, M.; Dodin, S. Blood pressure and endothelial function in healthy, pregnant women after acute and daily consumption of flavanol-rich chocolate: A pilot, randomized controlled trial. Nutr. J. 2013, 12, 41. [CrossRef] [PubMed]

91. Monagas, M.; Khan, N.; Andres-Lacueva, C.; Casas, R.; Urpi-Sarda, M.; Llorach, R.; Lamuela-Raventos, R.M.; Estruch, R. Effect of cocoa powder on the modulation of inflammatory biomarkers in patients at high risk of cardiovascular disease. Am. J. Clin. Nutr. 2009, 90, 1144-1150. [CrossRef] [PubMed]

92. Monahan, K.D.; Feehan, R.P.; Kunselman, A.R.; Preston, A.G.; Miller, D.L.; Lott, M.E.J. Dose-dependent increases in flow-mediated dilation following acute cocoa ingestion in healthy older adults. J. Appl. Physiol. 2011, 111, 1568-1574. [CrossRef] [PubMed]

93. Mousavi, A.; Vafa, M.; Neyestani, T.; Khamseh, M.; Hoseini, F. The effects of green tea consumption on metabolic and anthropometric indices in patients with type 2 diabetes. J. Res. Med. Sci. 2013, 18, 1080-1086. [PubMed]

94. Mozaffari-Khosravi, H.; Jalali-Khanabadi, B.A.; Afkhami-Ardekani, M.; Fatehi, F. Effects of sour tea (hibiscus sabdariffa) on lipid profile and lipoproteins in patients with type ii diabetes. J. Altern. Complement. Med. 2009, 15, 899-903. [CrossRef] [PubMed]

95. Muniyappa, R.; Hall, G.; Kolodziej, T.L.; Karne, R.J.; Crandon, S.K.; Quon, M.J. Cocoa consumption for 2 wk enhances insulin-mediated vasodilatation without improving blood pressure or insulin resistance in essential hypertension. Am. J. Clin. Nutr. 2008, 88, 1685-1696. [CrossRef] [PubMed] 
96. Murphy, K.J.; Chronopoulos, A.K.; Singh, I.; Francis, M.A.; Moriarty, H.; Pike, M.J.; Turner, A.H.; Mann, N.J.; Sinclair, A.J. Dietary flavanols and procyanidin oligomers from cocoa (theobroma cacao) inhibit platelet function. Am. J. Clin. Nutr. 2003, 77, 1466-1473. [PubMed]

97. Nagao, T.; Hase, T.; Tokimitsu, I. A green tea extract high in catechins reduces body fat and cardiovascular risks in humans. Obes. Silver Spring 2007, 15, 1473-1483. [CrossRef] [PubMed]

98. Nagao, T.; Komine, Y.; Soga, S.; Meguro, S.; Hase, T.; Tanaka, Y.; Tokimitsu, I. Ingestion of a tea rich in catechins leads to a reduction in body fat and malondialdehyde-modified LDL in men. Am. J. Clin. Nutr. 2005, 81, 122-129. [PubMed]

99. Nagao, T.; Meguro, S.; Hase, T.; Otsuka, K.; Komikado, M.; Tokimitsu, I.; Yamamoto, T.; Yamamoto, K. A catechin-rich beverage improves obesity and blood glucose control in patients with type 2 diabetes. Obes. Silver Spring 2009, 17, 310-317. [CrossRef] [PubMed]

100. Nagasako-Akazome, Y.; Kanda, T.; Ohtake, Y.; Shimasaki, H.; Kobayashi, T. Apple polyphenols influence cholesterol metabolism in healthy subjects with relatively high body mass index. J. Oleo Sci. 2007, 56, 417-428. [CrossRef] [PubMed]

101. Nantz, M.P.; Rowe, C.A.; Bukowski, J.F.; Percival, S.S. Standardized capsule of camellia sinensis lowers cardiovascular risk factors in a randomized, double-blind, placebo-controlled study. Nutrition 2009, 25, 147-154. [CrossRef]

102. Nickols-Richardson, S.M.; Piehowski, K.E.; Metzgar, C.J.; Miller, D.L.; Preston, A.G. Changes in body weight, blood pressure and selected metabolic biomarkers with an energy-restricted diet including twice daily sweet snacks and once daily sugar-free beverage. Nutr. Res. Pract. 2014, 8, 695-704. [CrossRef] [PubMed]

103. Njike, V.Y.; Faridi, Z.; Shuval, K.; Dutta, S.; Kay, C.D.; West, S.G.; Kris-Etherton, P.M.; Katz, D.L. Effects of sugar-sweetened and sugar-free cocoa on endothelial function in overweight adults. Int. J. Cardiol. 2011, 149, 83-88. [CrossRef] [PubMed]

104. Oi, Y.; Fujita, H. Body weight and body mass index (bmi) in preobese and overweight japanese adults treated with black chinese tea water extract (bte) using a more appropriate statistical analysis method. Int. J. Food Prop. 2015, 18, 1345-1349. [CrossRef]

105. Osakabe, N.; Baba, S.; Yasuda, A.; Iwamoto, T.; Kamiyama, M.; Tokunaga, T.; Kondo, K. Dose-response study of daily cocoa intake on the oxidative susceptibility of low-density lipoprotein in healthy human volunteers. J. Health Sci. 2004, 50, 679-684. [CrossRef]

106. Parsaeyan, N.; Mozaffari-Khosravi, H.; Absalan, A.; Mozayan, M.R. Beneficial effects of cocoa on lipid peroxidation and inflammatory markers in type 2 diabetic patients and investigation of probable interactions of cocoa active ingredients with prostaglandin synthase-2 (ptgs-2/cox-2) using virtual analysis. J. Diabetes Metab. Disord. 2014, 13, 30. [CrossRef] [PubMed]

107. Ravn-Haren, G.; Dragsted, L.O.; Buch-Andersen, T.; Jensen, E.N.; Jensen, R.I.; Nemeth-Balogh, M.; Paulovicsova, B.; Bergstrom, A.; Wilcks, A.; Licht, T.R.; et al. Intake of whole apples or clear apple juice has contrasting effects on plasma lipids in healthy volunteers. Eur. J. Nutr. 2013, 52, 1875-1889. [CrossRef] [PubMed]

108. Rostami, A.; Khalili, M.; Haghighat, N.; Eghtesadi, S.; Shidfar, F.; Heidari, I.; Ebrahimpour-Koujan, S.; Eghtesadi, M. High-cocoa polyphenol-rich chocolate improves blood pressure in patients with diabetes and hypertension. ARYA Atheroscler. 2015, 11, 21-29. [PubMed]

109. Rull, G.; Mohd-Zain, Z.N.; Shiel, J.; Lundberg, M.H.; Collier, D.J.; Johnston, A.; Warner, T.D.; Corder, R. Effects of high flavanol dark chocolate on cardiovascular function and platelet aggregation. Vasc. Pharmacol. 2015, 71, 70-78. [CrossRef] [PubMed]

110. Ryu, O.H.; Lee, J.; Lee, K.W.; Kim, H.Y.; Seo, J.A.; Kim, S.G.; Kim, N.H.; Baik, S.H.; Choi, D.S.; Choi, K.M. Effects of green tea consumption on inflammation, insulin resistance and pulse wave velocity in type 2 diabetes patients. Diabetes Res. Clin. Pract. 2006, 71, 356-358. [CrossRef] [PubMed]

111. Sansone, R.; Rodriguez-Mateos, A.; Heuel, J.; Falk, D.; Schuler, D.; Wagstaff, R.; Kuhnle, G.G.; Spencer, J.P.; Schroeter, H.; Merx, M.W.; et al. Cocoa flavanol intake improves endothelial function and framingham risk score in healthy men and women: A randomised, controlled, double-masked trial: The flaviola health study. Br. J. Nutr. 2015, 114, 1246-1255. [CrossRef] [PubMed] 
112. Shiina, Y.; Funabashi, N.; Lee, K.; Murayama, T.; Nakamura, K.; Wakatsuki, Y.; Daimon, M.; Komuro, I. Acute effect of oral flavonoid-rich dark chocolate intake on coronary circulation, as compared with non-flavonoid white chocolate, by transthoracic doppler echocardiography in healthy adults. Int. J. Cardiol. 2009, 131, 424-429. [CrossRef] [PubMed]

113. Shimada, K.; Kawarabayashi, T.; Tanaka, A.; Fukuda, D.; Nakamura, Y.; Yoshiyama, M.; Takeuchi, K.; Sawaki, T.; Hosoda, K.; Yoshikawa, J. Oolong tea increases plasma adiponectin levels and low-density lipoprotein particle size in patients with coronary artery disease. Diabetes Res. Clin. Pract. 2004, 65, 227-234. [CrossRef] [PubMed]

114. Sone, T.; Kuriyama, S.; Nakaya, N.; Hozawa, A.; Shimazu, T.; Nomura, K.; Rikimaru, S.; Tsuji, I. Randomized controlled trial for an effect of catechin-enriched green tea consumption on adiponectin and cardiovascular disease risk factors. Food Nutr. Res. 2011, 55. [CrossRef] [PubMed]

115. Stendell-Hollis, N.R.; Thomson, C.A.; Thompson, P.A.; Bea, J.W.; Cussler, E.C.; Hakim, I.A. Green tea improves metabolic biomarkers, not weight or body composition: A pilot study in overweight breast cancer survivors. J. Hum. Nutr. Diet. 2010, 23, 590-600. [CrossRef] [PubMed]

116. Suliburska, J.; Bogdanski, P.; Szulinska, M.; Stepien, M.; Pupek-Musialik, D.; Jablecka, A. Effects of green tea supplementation on elements, total antioxidants, lipids, and glucose values in the serum of obese patients. Biol. Trace Elem. Res. 2012, 149, 315-322. [CrossRef] [PubMed]

117. Takahashi, M.; Miyashita, M.; Suzuki, K.; Bae, S.R.; Kim, H.K.; Wakisaka, T.; Matsui, Y.; Takeshita, M.; Yasunaga, K. Acute ingestion of catechin-rich green tea improves postprandial glucose status and increases serum thioredoxin concentrations in postmenopausal women. Br. J. Nutr. 2014, 112, 1542-1550. [CrossRef] [PubMed]

118. Taubert, D.; Roesen, R.; Lehmann, C.; Jung, N.; Schomig, E. Effects of low habitual cocoa intake on blood pressure and bioactive nitric oxide: A randomized controlled trial. JAMA 2007, 298, 49-60. [CrossRef] [PubMed]

119. Tinahones, F.J.; Rubio, M.A.; Garrido-Sanchez, L.; Ruiz, C.; Gordillo, E.; Cabrerizo, L.; Cardona, F. Green tea reduces LDL oxidability and improves vascular function. J. Am. Coll. Nutr. 2008, 27, 209-213. [CrossRef] [PubMed]

120. Toolsee, N.A.; Aruoma, O.I.; Gunness, T.K.; Kowlessur, S.; Dambala, V.; Murad, F.; Googoolye, K.; Daus, D.; Indelicato, J.; Rondeau, P.; et al. Effectiveness of green tea in a randomized human cohort: Relevance to diabetes and its complications. Biomed. Res. Int. 2013, 2013, 412379. [CrossRef] [PubMed]

121. Troup, R.; Hayes, J.H.; Raatz, S.K.; Thyagarajan, B.; Khaliq, W.; Jacobs, D.R.; Key, N.S.; Morawski, B.M.; Kaiser, D.; Bank, A.J.; et al. Effect of black tea intake on blood cholesterol concentrations in individuals with mild hypercholesterolemia: A diet-controlled randomized trial. J. Acad. Nutr. Diet. 2015, 115, 264-271. [CrossRef] [PubMed]

122. Tsai Ch, H.; Chiu, W.C.; Yang, N.C.; Ouyang, C.M.; Yen, Y.H. A novel green tea meal replacement formula for weight loss among obese individuals: A randomized controlled clinical trial. Int. J. Food Sci. Nutr. 2009, 60 (Suppl. 6), 151-159. [CrossRef] [PubMed]

123. Tzounis, X.; Rodriguez-Mateos, A.; Vulevic, J.; Gibson, G.R.; Kwik-Uribe, C.; Spencer, J.P. Prebiotic evaluation of cocoa-derived flavanols in healthy humans by using a randomized, controlled, double-blind, crossover intervention study. Am. J. Clin. Nutr. 2011, 93, 62-72. [CrossRef] [PubMed]

124. Unno, T.; Tago, M.; Suzuki, Y.; Nozawa, A.; Sagesaka, Y.M.; Kakuda, T.; Egawa, K.; Kondo, K. Effect of tea catechins on postprandial plasma lipid responses in human subjects. Br. J. Nutr. 2005, 93, 543-547. [CrossRef] [PubMed]

125. Vafa, M.R.; Haghighatjoo, E.; Shidfar, F.; Afshari, S.; Gohari, M.R.; Ziaee, A. Effects of apple consumption on lipid profile of hyperlipidemic and overweight men. Int. J. Prev. Med. 2011, 2, 94-100. [PubMed]

126. Velliquette, R.A.; Grann, K.; Missler, S.R.; Patterson, J.; Hu, C.; Gellenbeck, K.W.; Scholten, J.D.; Randolph, R.K. Identification of a botanical inhibitor of intestinal diacylglyceride acyltransferase 1 activity via in vitro screening and a parallel, randomized, blinded, placebo-controlled clinical trial. Nutr. Metab. 2015, 12, 27. [CrossRef] [PubMed]

127. Vieira Senger, A.E.; Schwanke, C.H.; Gomes, I.; Valle Gottlieb, M.G. Effect of green tea (camellia sinensis) consumption on the components of metabolic syndrome in elderly. J. Nutr. Health Aging 2012, 16, 738-742. [CrossRef] [PubMed] 
128. Villaño, D.; Pecorari, M.; Testa, M.F.; Raguzzini, A.; Stalmach, A.; Crozier, A.; Tubili, C.; Serafini, M. Unfermented and fermented rooibos teas (aspalathus linearis) increase plasma total antioxidant capacity in healthy humans. Food Chem. 2010, 123, 679-683. [CrossRef]

129. Vlachopoulos, C.; Aznaouridis, K.; Alexopoulos, N.; Economou, E.; Andreadou, I.; Stefanadis, C. Effect of dark chocolate on arterial function in healthy individuals. Am. J. Hypertens. 2005, 18, 785-791. [CrossRef] [PubMed]

130. Wang, H.; Wen, Y.; Du, Y.; Yan, X.; Guo, H.; Rycroft, J.A.; Boon, N.; Kovacs, E.M.; Mela, D.J. Effects of catechin enriched green tea on body composition. Obes. Silver Spring 2010, 18, 773-779. [CrossRef] [PubMed]

131. West, S.G.; McIntyre, M.D.; Piotrowski, M.J.; Poupin, N.; Miller, D.L.; Preston, A.G.; Wagner, P.; Groves, L.F.; Skulas-Ray, A.C. Effects of dark chocolate and cocoa consumption on endothelial function and arterial stiffness in overweight adults. Br. J. Nutr. 2014, 111, 653-661. [CrossRef] [PubMed]

132. Westphal, S.; Luley, C. Flavanol-rich cocoa ameliorates lipemia-induced endothelial dysfunction. Heart Vessel. 2011, 26, 511-515. [CrossRef] [PubMed]

133. Widlansky, M.E.; Hamburg, N.M.; Anter, E.; Holbrook, M.; Kahn, D.F.; Elliott, J.G.; Keaney, J.F., Jr.; Vita, J.A. Acute egcg supplementation reverses endothelial dysfunction in patients with coronary artery disease. J. Am. Coll. Nutr. 2007, 26, 95-102. [CrossRef] [PubMed]

134. Widmer, R.J.; Freund, M.A.; Flammer, A.J.; Sexton, J.; Lennon, R.; Romani, A.; Mulinacci, N.; Vinceri, F.F.; Lerman, L.O.; Lerman, A. Beneficial effects of polyphenol-rich olive oil in patients with early atherosclerosis. Eur. J. Nutr. 2013, 52, 1223-1231. [CrossRef] [PubMed]

135. Wu, A.H.; Spicer, D.; Stanczyk, F.Z.; Tseng, C.C.; Yang, C.S.; Pike, M.C. Effect of 2-month controlled green tea intervention on lipoprotein cholesterol, glucose, and hormone levels in healthy postmenopausal women. Cancer Prev. Res. 2012, 5, 393-402. [CrossRef] [PubMed]

136. Yang, H.Y.; Yang, S.C.; Chao, J.C.; Chen, J.R. Beneficial effects of catechin-rich green tea and inulin on the body composition of overweight adults. Br. J. Nutr. 2012, 107, 749-754. [CrossRef] [PubMed]

137. Yang, T.Y.; Chou, J.I.; Ueng, K.C.; Chou, M.Y.; Yang, J.J.; Lin-Shiau, S.Y.; Hu, M.E.; Lin, J.K. Weight reduction effect of puerh tea in male patients with metabolic syndrome. Phytother. Res. 2014, 28, 1096-1101. [CrossRef] [PubMed]

138. Li, Y.; Wang, C.; Huai, Q.; Guo, F.; Liu, L.; Feng, R.; Sun, C. Effects of tea or tea extract on metabolic profiles in patients with type 2 diabetes mellitus: A meta-analysis of ten randomized controlled trials. Diabetes Metab. Res. Rev. 2016, 32, 2-10. [CrossRef] [PubMed]

139. Zhao, Y.; Asimi, S.; Wu, K.; Zheng, J.; Li, D. Black tea consumption and serum cholesterol concentration: Systematic review and meta-analysis of randomized controlled trials. Clin. Nutr. 2015, 34, 612-619. [CrossRef] [PubMed]

140. Zhong, X.; Zhang, T.; Liu, Y.; Wei, X.; Zhang, X.; Qin, Y.; Jin, Z.; Chen, Q.; Ma, X.; Wang, R.; et al. Short-term weight-centric effects of tea or tea extract in patients with metabolic syndrome: A meta-analysis of randomized controlled trials. Nutr. Diabetes 2015, 5, e160. [CrossRef] [PubMed]

141. Wang, D.; Chen, C.; Wang, Y.; Liu, J.; Lin, R. Effect of black tea consumption on blood cholesterol: A meta-analysis of 15 randomized controlled trials. PLOS ONE 2014, 9, e107711. [CrossRef] [PubMed]

142. Onakpoya, I.; Spencer, E.; Heneghan, C.; Thompson, M. The effect of green tea on blood pressure and lipid profile: A systematic review and meta-analysis of randomized clinical trials. Nutr. Metab. Cardiovasc. Dis. 2014, 24, 823-836. [CrossRef] [PubMed]

143. Baladia, E.; Basulto, J.; Manera, M.; Martínez, R.; Calbet, D. Effect of green tea or green tea extract consumption on body weight and body composition; systematic review and meta-analysis. Nutr. Hosp. 2014, 29, 479-490. [PubMed]

144. Khalesi, S.; Sun, J.; Buys, N.; Jamshidi, A.; Nikbakht-Nasrabadi, E.; Khosravi-Boroujeni, H. Green tea catechins and blood pressure: A systematic review and meta-analysis of randomised controlled trials. Eur. J. Nutr. 2014, 53, 1299-1311. [CrossRef] [PubMed]

145. Hartley, L.; Flowers, N.; Holmes, J.; Clarke, A.; Stranges, S.; Hooper, L.; Rees, K. Green and black tea for the primary prevention of cardiovascular disease. Cochrane Database Syst. Rev. 2013, CD009934. [CrossRef]

146. Kim, A.; Chiu, A.; Barone, M.K.; Avino, D.; Wang, F.; Coleman, C.I.; Phung, O.J. Green tea catechins decrease total and low-density lipoprotein cholesterol: A systematic review and meta-analysis. J. Am. Diet. Assoc. 2011, 111, 1720-1729. [CrossRef] [PubMed] 
147. Zheng, X.X.; Xu, Y.L.; Li, S.H.; Liu, X.X.; Hui, R.; Huang, X.H. Green tea intake lowers fasting serum total and LDL cholesterol in adults: A meta-analysis of 14 randomized controlled trials. Am. J. Clin. Nutr. 2011, 94, 601-610. [CrossRef] [PubMed]

148. Phung, O.J.; Baker, W.L.; Matthews, L.J.; Lanosa, M.; Thorne, A.; Coleman, C.I. Effect of green tea catechins with or without caffeine on anthropometric measures: A systematic review and meta-analysis. Am. J. Clin. Nutr. 2010, 91, 73-81. [CrossRef] [PubMed]

149. Tokede, O.A.; Gaziano, J.M.; Djousse, L. Effects of cocoa products/dark chocolate on serum lipids: A meta-analysis. Eur. J. Clin. Nutr. 2011, 65, 879-886. [CrossRef] [PubMed]

150. Lin, X.; Zhang, I.; Li, A.; Manson, J.E.; Sesso, H.D.; Wang, L.; Liu, S. Cocoa Flavanol Intake and Biomarkers for Cardiometabolic Health: A Systematic Review and Meta-Analysis of Randomized Controlled Trials. J. Nutr. 2016, 146, 2325-2333. [CrossRef] [PubMed]

151. Hooper, L.; Kay, C.; Abdelhamid, A.; Kroon, P.A.; Cohn, J.S.; Rimm, E.B.; Cassidy, A. Effects of chocolate, cocoa, and flavan-3-ols on cardiovascular health: A systematic review and meta-analysis of randomized trials. Am. J. Clin. Nutr. 2012, 95, 740-751. [CrossRef] [PubMed]

152. De Pascual-Teresa, S.; Santos-Buelga, C.; Rivas-Gonzalo, J.C. Quantitative analysis of flavan-3-ols in Spanish foodstuffs and beverages. J. Agric. Food. Chem. 2000, 48, 5331-5337. [CrossRef] [PubMed]

153. Hendijani, F.; Akbari, V. Probiotic supplementation for management of cardiovascular risk factors in adults with type ii diabetes: A systematic review and meta-analysis. Clin. Nutr. 2017. [CrossRef] [PubMed]

154. Zhang, Q.; Wu, Y.; Fei, X. Effect of probiotics on body weight and body-mass index: A systematic review and meta-analysis of randomized, controlled trials. Int. J. Food. Sci. Nutr. 2016, 67, 571-580. [CrossRef] [PubMed]

155. Sugiyama, M.G.; Agellon, L.B. Sex differences in lipid metabolism and metabolic disease risk. Biochem. Cell Biol. 2012, 90, 124-141. [CrossRef] [PubMed]

156. Wahi, G.; Anand, S.S. Race/ethnicity, obesity, and related cardio-metabolic risk factors: A life-course perspective. Curr. Cardiovasc. Risk Rep. 2013, 7, 326-335. [CrossRef] [PubMed]

157. Chen, C.; Yu, X.; Shao, S. Effects of omega-3 fatty acid supplementation on glucose control and lipid levels in type 2 diabetes: A meta-analysis. PLoS ONE 2015, 10, e0139565. [CrossRef] [PubMed]

158. Pereira, T.M.; Pimenta, F.S.; Porto, M.L.; Baldo, M.P.; Campagnaro, B.P.; Gava, A.L.; Meyrelles, S.S.; Vasquez, E.C. Coadjuvants in the diabetic complications: Nutraceuticals and drugs with pleiotropic effects. Int. J. Mol. Sci. 2016, 17, 1273. [CrossRef] [PubMed]

159. Cohen, J. Statistical Power Analysis for the Behavioral Sciences, 2nd ed.; Erlbaum: Hillsdale, NJ, USA, 1988.

160. Stelmach-Mardas, M.; Walkowiak, J. Dietary interventions and changes in cardio-metabolic parameters in metabolically healthy obese subjects: A systematic review with meta-analysis. Nutrients 2016, 8, 455. [CrossRef] [PubMed]

161. Mead, E.; Atkinson, G.; Richter, B.; Metzendorf, M.I.; Baur, L.; Finer, N.; Corpeleijn, E.; O’Malley, C.; Ells, L.J. Drug interventions for the treatment of obesity in children and adolescents. Cochrane Database Syst. Rev. 2016, 11, CD012436. [PubMed]

162. Mabire, L.; Mani, R.; Liu, L.; Mulligan, H.; Baxter, D. The influence of age, sex and body mass index on the effectiveness of brisk walking for obesity management in adults: A systematic review and meta-analysis. J. Phys. Act. Health 2017, 14, 389-407. [CrossRef] [PubMed]

163. Peirson, L.; Fitzpatrick-Lewis, D.; Morrison, K.; Warren, R.; Usman Ali, M.; Raina, P. Treatment of overweight and obesity in children and youth: A systematic review and meta-analysis. CMAJ Open 2015, 3, E35-E46. [CrossRef] [PubMed]

164. Kucukgoncu, S.; Zhou, E.; Lucas, K.B.; Tek, C. Alpha-lipoic acid (ala) as a supplementation for weight loss: Results from a meta-analysis of randomized controlled trials. Obes. Rev. 2017, 18, 594-601. [CrossRef] [PubMed]

165. Zhang, Y.Y.; Liu, W.; Zhao, T.Y.; Tian, H.M. Efficacy of omega-3 polyunsaturated fatty acids supplementation in managing overweight and obesity: A meta-analysis of randomized clinical trials. J. Nutr. Health Aging 2017, 21, 187-192. [CrossRef] [PubMed]

166. Taylor, F.; Huffman, M.D.; Macedo, A.F.; Moore, T.H.; Burke, M.; Davey Smith, G.; Ward, K.; Ebrahim, S. Statins for the primary prevention of cardiovascular disease. Cochrane Database Syst. Rev. 2013, CD004816. [CrossRef] 
167. Sahebkar, A.; Serban, M.C.; Gluba-Brzozka, A.; Mikhailidis, D.P.; Cicero, A.F.; Rysz, J.; Banach, M. Lipid-modifying effects of nutraceuticals: An evidence-based approach. Nutrition 2016, 32, 1179-1192. [CrossRef] [PubMed]

168. Silverman, M.G.; Ference, B.A.; Im, K.; Wiviott, S.D.; Giugliano, R.P.; Grundy, S.M.; Braunwald, E.; Sabatine, M.S. Association between lowering LDL-C and cardiovascular risk reduction among different therapeutic interventions: A systematic review and meta-analysis. JAMA 2016, 316, 1289-1297. [CrossRef] [PubMed]

169. Samavat, H.; Newman, A.R.; Wang, R.; Yuan, J.M.; Wu, A.H.; Kurzer, M.S. Effects of green tea catechin extract on serum lipids in postmenopausal women: A randomized, placebo-controlled clinical trial. Am. J. Clin. Nutr. 2016, 104, 1671-1682. [CrossRef] [PubMed]

170. Taub, P.R.; Ramirez-Sanchez, I.; Patel, M.; Higginbotham, E.; Moreno-Ulloa, A.; Roman-Pintos, L.M.; Phillips, P.; Perkins, G.; Ceballos, G.; Villarreal, F. Beneficial effects of dark chocolate on exercise capacity in sedentary subjects: Underlying mechanisms. A double blind, randomized, placebo controlled trial. Food Funct. 2016, 7, 3686-3693. [CrossRef] [PubMed]

171. Lu, P.H.; Hsu, C.H. Does supplementation with green tea extract improve acne in post-adolescent women? A randomized, double-blind, and placebo-controlled clinical trial. Complement. Ther. Med. 2016, 25, 159-163. [CrossRef] [PubMed]

172. Dostal, A.M.; Arikawa, A.; Espejo, L.; Kurzer, M.S. Long-term supplementation of green tea extract does not modify adiposity or bone mineral density in a randomized trial of overweight and obese postmenopausal women. J. Nutr. 2016, 146, 256-264. [CrossRef] [PubMed]

173. Most, J.; van Can, J.G.; van Dijk, J.W.; Goossens, G.H.; Jocken, J.; Hospers, J.J.; Bendik, I.; Blaak, E.E. A 3-day egcg-supplementation reduces interstitial lactate concentration in skeletal muscle of overweight subjects. Sci. Rep. 2015, 5, 17896. [CrossRef] [PubMed]

174. Dostal, A.M.; Samavat, H.; Espejo, L.; Arikawa, A.Y.; Stendell-Hollis, N.R.; Kurzer, M.S. Green tea extract and catechol-o-methyltransferase genotype modify fasting serum insulin and plasma adiponectin concentrations in a randomized controlled trial of overweight and obese postmenopausal women. J. Nutr. 2016, 146, 38-45. [CrossRef] [PubMed]

175. Basu, A.; Betts, N.M.; Leyva, M.J.; Fu, D.; Aston, C.E.; Lyons, T.J. Acute cocoa supplementation increases postprandial HDL cholesterol and insulin in obese adults with type 2 diabetes after consumption of a high-fat breakfast. J. Nutr. 2015, 145, 2325-2332. [CrossRef] [PubMed]

176. Sarria, B.; Martinez-Lopez, S.; Sierra-Cinos, J.L.; Garcia-Diz, L.; Goya, L.; Mateos, R.; Bravo, L. Effects of bioactive constituents in functional cocoa products on cardiovascular health in humans. Food Chem. 2015, 174, 214-218. [CrossRef] [PubMed]

177. Gutierrez-Salmean, G.; Meaney, E.; Lanaspa, M.A.; Cicerchi, C.; Johnson, R.J.; Dugar, S.; Taub, P.; Ramirez-Sanchez, I.; Villarreal, F.; Schreiner, G.; et al. A randomized, placebo-controlled, double-blind study on the effects of (-)-epicatechin on the triglyceride/HDLC ratio and cardiometabolic profile of subjects with hypertriglyceridemia: Unique in vitro effects. Int. J. Cardiol. 2016, 223, 500-506. [CrossRef] [PubMed]

178. Imbe, H.; Sano, H.; Miyawaki, M.; Fujisawa, R.; Miyasato, M.; Nakatsuji, F.; Haseda, F.; Tanimoto, K.; Terasaki, J.; Maeda-Yamamoto, M.; et al. “Benifuuki” green tea, containing o-methylated egcg, reduces serum low-density lipoprotein cholesterol and lectin-like oxidized low-density lipoprotein receptor-1 ligands containing apolipoprotein b: A double-blind, placebo-controlled randomized trial. J. Funct. Foods 2016, 25, 25-37. [CrossRef]

179. Lee, T.M.; Charng, M.J.; Tseng, C.D.; Lai, L.P. A double-blind, randomized, placebo-controlled study to evaluate the efficacy and safety of sta-2 (green tea polyphenols) in patients with chronic stable angina. Acta Cardiol. Sin. 2016, 32, 439-449. [PubMed]

180. Ide, K.; Yamada, H.; Takuma, N.; Kawasaki, Y.; Harada, S.; Nakase, J.; Ukawa, Y.; Sagesaka, Y.M. Effects of green tea consumption on cognitive dysfunction in an elderly population: A randomized placebo-controlled study. Nutr. J. 2016, 15, 49. [CrossRef] [PubMed]

181. Mahmoud, F.; Haines, D.; Al-Ozairi, E.; Dashti, A. Effect of black tea consumption on intracellular cytokines, regulatory $t$ cells and metabolic biomarkers in type 2 diabetes patients. Phytother. Res. 2016, 30, 454-462. [CrossRef] [PubMed] 
182. Ibero-Baraibar, I.; Suarez, M.; Arola-Arnal, A.; Zulet, M.A.; Martinez, J.A. Cocoa extract intake for 4 weeks reduces postprandial systolic blood pressure response of obese subjects, even after following an energy-restricted diet. Food Nutr. Res. 2016, 60, 30449. [CrossRef] [PubMed]

183. Kobayashi, M.; Kawano, T.; Ukawa, Y.; Sagesaka, Y.M.; Fukuhara, I. Green tea beverages enriched with catechins with a galloyl moiety reduce body fat in moderately obese adults: A randomized double-blind placebo-controlled trial. Food Funct. 2016, 7, 498-507. [CrossRef] [PubMed]

(C) 2017 by the authors. Licensee MDPI, Basel, Switzerland. This article is an open access article distributed under the terms and conditions of the Creative Commons Attribution (CC BY) license (http:/ / creativecommons.org/licenses/by/4.0/). 